\title{
Near-infrared spectroscopy of AGB star candidates in Fornax, Sculptor, and NGC 6822 $\star, \star \star$
}

\author{
M. A. T. Groenewegen ${ }^{1}$, A. Lançon ${ }^{2}$, and M. Marescaux ${ }^{3}$
}

\author{
${ }^{1}$ Koninklijke Sterrenwacht van België, Ringlaan 3, 1180 Brussels, Belgium \\ e-mail: marting@oma.be \\ 2 Observatoire Astronomique, Université de Strasbourg \& CNRS (UMR 7550), 11 rue de l'Université, 67000 Strasbourg, France \\ 3 Instituut voor Sterrenkunde, Katholieke Universiteit Leuven, Celestijnenlaan 200 D, 3001 Leuven, Belgium
}

Received 16 January 2009 / Accepted 22 July 2009

\section{ABSTRACT}

\begin{abstract}
Context. The asymptotic giant branch (AGB) phase is characterised by substantial mass loss that is accompanied by the formation of dust. In extreme cases this will make the star no longer visible in the optical. For a better understanding of AGB evolution it is important to identify and characterise these very red AGB stars.

Aims. The first aim of this article is to improve the census of red AGB stars in three Local Group galaxies, based on near-IR spectroscopic observations of new candidates with red 2MASS $(J-K)$ colours. The opportunity is taken to compare the near-IR spectra with those of Milky Way stars.

Methods. We used ISAAC on the ESO VLT to take $J$ and $H$-band spectra of 36 targets in Fornax, Sculptor and NGC 6822.

Results. Twelve new C-stars are found in Fornax, and one is confirmed in Sculptor. All C-stars have $(J-K)>1.6$, and are brighter than -3.55 in bolometric magnitude. Ten new oxygen-rich late-type giant stars are identified in Fornax, but none is extremely red or very luminous. Five luminous O-rich AGB stars are identified in NGC 6822, of which 3 show water absorption, indicative of spectral type M. Again, none is as red as Milky Way OH/IR stars, but in this galaxy the list of candidate AGB stars is biased against very red objects. In some C-stars with $(J-K)>2$ an extremely strong $1.53 \mu \mathrm{m}$ absorption band is found. These stars are probably all Mira variables and the feature is related to the low temperature, high density chemistry that is a first step towards dust formation and mass loss.
\end{abstract}

Key words. stars: AGB and post-AGB - stars: carbon - galaxies: Local Group

\section{Introduction}

Asymptotic giant branch (AGB) stars play an important role in stellar population studies (e.g. Lançon 1999) and galactic structure studies (e.g. Dejonghe \& Caelenberg 1999), and through their mass loss they contribute significantly to the enrichment of the interstellar medium (Habing 1996; see Kerschbaum et al. 2007, for recent reviews). The AGB phase is the final evolutionary stage for more than $95 \%$ of all stars that leave the main sequence (MS) in a Hubble time. Their intrinsically high luminosities and well defined evolutionary stage make them important constituents and probes of extragalactic systems. Due to their old age they define highly relaxed subsystems. At the same time, the extragalactic studies are important for our understanding of AGB-evolution itself.

Even though the AGB evolution is relatively well understood, there remain a number of important unsolved questions, such as the MS mass interval for which carbon star formation is possible (as a function of metallicity), or the mass loss dependence on time, mass, metallicity. By observing AGB populations at known distances and of different metallicity light can be shed on these and other related questions.

\footnotetext{
* Based on observations collected at the European Southern Observatory, Paranal, Chile (ESO programme 75.D-0152).

$\star \star$ Figure 6 is only available in electronic form via

http: //www . aanda.org
}

One of the most efficient methods for the identification of AGB stars in extragalactic systems uses two narrow-band filters at 7800 and $8100 \AA$, centred on a CN-band in carbon stars (and near-continuum in oxygen-rich stars), and a $\mathrm{TiO}$ band in oxygen-rich stars (and continuum in C-stars), respectively, and two broad-band filters from the set $V, R, I$. In an [78-81] versus $[V-I]$ (or $[R-I]$ ) colour-colour plot, carbon stars and late-type oxygen-rich stars clearly separate redwards of $(V-I) \approx 1$.6. For an illustration of this, see Cook \& Aaronson (1989) or Nowotny \& Kerschbaum (2002). This narrow-band filter system was introduced by Wing (1971) and Palmer \& Wing (1982) and then first applied by Richer et al. (1984) and Aaronson et al. (1984). In recent years several groups have used this technique to survey many galaxies of the Local Group (LG), see e.g., Battinelli $\&$ Demers (2005a,b) for a summary of results of their surveys of more than $10 \mathrm{LG}$ galaxies over the last 6 years, Rowe et al. (2005), Nowotny et al. (2001, 2003) or Harbeck et al. (2004).

The narrow-band surveys identify AGB stars in the optical. However, as evolution on the AGB proceeds, the mass loss rate increases (culminating in the so called "superwind" phase) and hence the obscuration of the central star by dust formation becomes increasingly important. In fact, at some point, AGB stars become "invisible" in the optical. This phenomenon has been relatively well studied in the Galaxy, LMC, and SMC ever since it was first highlighted by the IRAS satellite, and with subsequent observation by the ISO satellite (e.g. Groenewegen 1995; Trams et al. 1999; Whitelock et al. 2003), and Spitzer (e.g. 
Groenewegen et al. 2007). However, in none of the other LG galaxies has this effect been properly studied, although Menzies et al. (2002) have discovered 5 very red objects in Leo I which they believe to be dust-enshrouded AGB stars, and recently other ground-based NIR studies have appeared (Cioni \& Habing 2005a,b; Davidge 2005; Kang et al. 2005, 2006; Sohn et al. 2006) that identify a candidate AGB star population in a few LG galaxies based on colours, and Matsuura et al. (2007) presented Spitzer IRS spectra for a few AGB stars in Fornax. The present paper tries to lift some of these optical biases by considering a sample based on 2MASS data, that will allow the detection of mass-losing stars.

In this paper we present NIR medium resolution spectroscopy of new AGB star candidates in the LG galaxies Fornax, Sculptor and NGC 6822, selected from 2MASS. Very few such spectra are available in the literature. In Sect. 2 the sample selection is outlined, and in Sect. 3 the colour-magnitude diagram is discussed. Section 4 discusses the observations, and Sect. 5 describes the spectra and classification. Section 6 presents a brief discussion of the stellar properties in the framework of the galaxy histories. We also highlight the behaviour of the interesting $1.53 \mu \mathrm{m}$ feature observed in the $H$-band spectra of some of the carbon stars.

\section{Sample selection}

The all-sky release of the 2MASS JHK survey (Cutri et al. 2003) offers a unique opportunity to detect the reddest AGB stars in Local Group galaxies. We searched the 2MASS point source catalog in the direction of all LG galaxies within $1 \mathrm{Mpc}$ of the Milky Way (excluding the SMC, the LMC, M 31, M 32 and M 33), and selected sources with de-reddened colour index $(J-K)_{0}>1.22$. This ensures that most carbon stars are selected. Also selected are unobscured O-rich AGB stars with spectral type $\sim$ M5 and later (Loup 2003, private communication based on the M-star models of Fluks et al. 1994), or reddened objects. Because spectral type changes rapidly over a small interval in $(J-K)$, uncertainties in the 2MASS colours are likely to lead to the inclusion of stars of earlier spectral types than M5 in the sample, especially near the completeness limit of 2MASS.

We further restricted the sample to objects fainter than $M_{\text {bol }}=-7$ in order to exclude foreground stars. For this purpose, first estimates of the bolometric luminosities were obtained using the bolometric correction at $K$ derived by Bessell \& Wood (1984) from star observations in 47 Tuc and the SMC. We rejected sources with errors larger than 0.12 mag on either $J$ or $K$, as well as objects listed as non-stellar in the SIMBAD database ${ }^{1}$. One of the main purposes of a first spectroscopic follow-up being the obtention of a more complete census of all the AGB stars in the target galaxies, we flagged spectroscopically confirmed AGB stars from the literature and kept only the remaining objects as new AGB star candidates. A total of 109 candidate AGB stars were found in 16 galaxies, as summarised in Groenewegen (2006).

Originally, spectroscopic observations were planned on the 34 AGB star candidates in Fornax only, but due to the high airmass of Fornax at the start of the scheduled nights we were allowed to observe the AGB star candidates in NGC 6822 and Sculptor. The AGB star candidates in these three galaxies and their near-IR magnitudes are listed in Table 1 with their 2MASS identifications. In the second column we list the "internal" names used hereafter in the figures and text.

\footnotetext{
${ }^{1}$ Operated at CDS, Centre de Données Astronomiques de Strasbourg.
}

In Table 1, bolometric magnitudes are also given. These values take the near-IR spectral classification of Sect. 4 into account. The relationship between bolometric correction and $(J-K)$ is taken from Bessell \& Wood (1984) for O-rich stars, and from Whitelock et al. (2006) for C-stars (the latter correction is systematically larger than the former by about $0.2 \mathrm{mag}$ ). To Fornax a distance modulus (DM) of $20.72 \pm 0.04$ (Rizzi 2007) is adopted, based on the TRGB, Red Clump (RC) and the Horizontal Branch (HB). To Sculptor a DM of 19.66 is adopted, the mean of $19.67 \pm 0.12$ based on RR Lyrae stars (Pietrzyński et al. 2008), $19.64 \pm 0.08$ based on the TRGB (Rizzi 2002) and $19.66 \pm 0.15$ from the HB (Rizzi 2002). To NGC 6822 a DM of 23.34 is adopted, the mean of $23.31 \pm 0.06$ based on Cepheids (Gieren et al. 2006), 23.36 \pm 0.17 based on RR Lyrae stars (Clementini et al. 2003), and 23.34 \pm 0.12 based on the TRGB (Cioni \& Habing 2005b). The photometry was dereddened using $A_{\mathrm{V}}=0.067$ (Fornax), 0.059 (Sculptor), 0.784 (NGC 6822) (Schlegel et al. 1998), and ratios $A_{\mathrm{J}} / A_{\mathrm{V}}=0.27$, and $A_{\mathrm{K}} / A_{\mathrm{V}}=0.11$.

\section{The colour-magnitude diagram}

Near-IR colour-magnitude diagrams are shown here for the target galaxies and the nearby field. The observations described in the next section lack the spectral resolution to determine radial velocities. The question whether a source is part of the galaxy or is a contaminant is therefore of concern.

Sources were extracted from the 2MASS catalog at the central position of the galaxies, and at two locations 1.5 degrees above and below in declination. Radii of $25^{\prime}, 20^{\prime}$ and $17.5^{\prime}$ were used respectively for Fornax, Sculptor and NGC 6822, both for the central and for the neighbouring positions (about 2-3 core radii following Mateo 1998), as used for the selection described in the previous section. Figure 1 shows the colour-magnitude diagrams for the central and the off-position (only every second star is shown in the combined diagram of the two off-positions for each galaxy). Stars with photometric errors above $0.12 \mathrm{mag}$ in either $J$ or $K$ are rejected.

Stars brighter than the tip of the RGB (TRGB) are those of interest in considering the AGB nature of the targets observed. The TRGB is located at $K=14.61 \pm 0.04$ (Gullieuszik et al. 2007) in Fornax, or $K_{0}=14.60$. In Sculptor it is located at $K_{0}=13.8$ (Babusiaux et al. 2005). In NGC 6822 it is found at $K=17.10 \pm 0.01$ (Cioni \& Habing 2005b), or about $K_{0}=17.0$. This implies a selection bias in the case of NGC 6822 as only the very brightest AGB stars can be observed given the limiting magnitude of $2 \mathrm{MASS}$.

Counting the number of sources brighter than the TRGB and redder than 1.22 in $(J-K)$, the statistical contamination is about $7 \%$ for Fornax (5/71). It is higher in NGC $6822(2 / 9)$ and basically undetermined in Sculptor (0/2).

\section{Observations}

The observations took place in visitor mode on the nights of 23-27 September 2005 using ISAAC (Moorwood et al. 1998) on ESO's VLT UT1 telescope.

Apart from the AGB star candidates we targeted a few latetype stars of known spectral type in Fornax and Sculptor (see Table 1 for the references), and 2 known M-dwarfs. The observations were taken in low-resolution mode (i.e. a resolution of about 500 for a $1^{\prime \prime}$ slit) centred at 1.25 and $1.65 \mu \mathrm{m}$, respectively, to cover the entire $J$ and $H$-band atmospheric windows. 
Table 1. AGB star candidates and targets observed.

\begin{tabular}{|c|c|c|c|c|c|c|c|}
\hline 2MASS identifier & $\begin{array}{l}\text { Other } \\
\text { identifier }\end{array}$ & $J$ & $H$ & $K$ & $M_{\mathrm{bol}}$ & $\begin{array}{r}\text { Spectral } \\
\text { type }\end{array}$ & Remarks; (references) \\
\hline 2MASS02380397-3420196 & Fornax1 & 15.764 & 14.836 & 14.499 & -3.24 & K3-K4: & \\
\hline 2MASS02401824-3428412 & Fornax2 & 15.914 & 15.136 & 14.542 & -3.10 & G8-K4: & \\
\hline 2MASS02401650-3415374 & Fornax3 & 16.122 & 15.137 & 14.853 & -2.88 & G8-K4: & \\
\hline 2MASS02414248-3421284 & Fornax 4 & 15.797 & 14.912 & 14.485 & -3.21 & $\mathrm{~K} 2-\mathrm{K} 3$ & \\
\hline 2MASS02400772-3425156 & Fornax5 & 16.195 & 15.460 & 14.845 & & - & not observed \\
\hline 2MASS02394347-3413379 & Fornax6 & 15.981 & 15.024 & 14.292 & & - & not a point source, no spectrum taken \\
\hline 2MASS02401171-3431187 & Fornax 7 & 16.027 & 15.131 & 14.658 & -2.99 & K2-K3 & \\
\hline 2MASS02403857-3428344 & Fornax8 & 15.789 & 14.813 & 14.551 & -3.21 & K3-K4: & \\
\hline 2MASS02405606-3431273 & Fornax9 & 15.917 & 15.064 & 14.541 & & - & not observed \\
\hline 2MASS02395732-3431211 & Fornax 10 & 15.907 & 15.095 & 14.666 & & - & not observed \\
\hline 2MASS02405333-3412130 & Fornax11 & 15.034 & 13.981 & 13.261 & -4.01 & $\mathrm{C}$ & 1.53 absent; (7) \\
\hline 2MASS02402188-3406309 & Fornax 12 & 15.729 & 14.843 & 14.474 & -3.27 & $\mathrm{~K} 3-\mathrm{K} 4$ & \\
\hline 2MASS02400666-3423222 & Fornax 13 & 14.485 & 13.377 & 12.618 & -4.63 & $\mathrm{C}$ & 1.53 weak; $(1,2,7)$ \\
\hline 2MASS02405288-3428303 & Fornax 14 & 15.706 & 14.784 & 14.343 & -3.31 & K2-K3 & \\
\hline 2MASS02400946-3406256 & Fornax 15 & 15.790 & 14.556 & 13.668 & -3.56 & $\mathrm{C}$ & 1.53 strong \\
\hline 2MASS02401349-3426192 & Fornax16 & 15.930 & 14.794 & 14.652 & -3.07 & $\operatorname{not} \mathrm{C}$ & $\mathrm{H}$ band not usable \\
\hline 2MASS02403123-3428441 & Fornax 17 & 14.745 & 13.689 & 13.072 & -4.23 & $\mathrm{C}$ & 1.53 weak; $(3,5,7)$ \\
\hline 2MASS02390184-3427528 & Fornax 18 & 15.672 & 14.987 & 14.302 & - & non stellar & 2MASX source \\
\hline 2MASS02405840-3408022 & Fornax19 & 15.994 & 15.030 & 14.591 & -3.03 & K2-K4 & \\
\hline 2MASS02401207-3426255 & Fornax 20 & 15.131 & 13.732 & 12.728 & -4.53 & $\mathrm{C}$ & 1.53 weak; (7) \\
\hline 2MASS02401778-3427357 & Fornax 21 & 15.424 & 14.122 & 13.182 & -4.05 & $\mathrm{C}$ & 1.53 weak; (7) \\
\hline 2MASS02400252-3403272 & Fornax 22 & 15.855 & 15.157 & 14.608 & -3.15 & $\mathrm{~K} 1-\mathrm{K} 2$ & \\
\hline 2MASS02404718-3438510 & Fornax 23 & 15.602 & 14.911 & 14.199 & - & non stellar & 2MASX source \\
\hline 2MASS02395421-3438368 & Fornax 24 & 15.601 & 14.162 & 13.167 & -4.09 & $\mathrm{C}$ & 1.53 weak; (7) \\
\hline 2MASS02391232-3432450 & Fornax 25 & 14.722 & 13.262 & 12.120 & -5.18 & $\mathrm{C}$ & very red, features weak, dust emission?; $(5,6,7)$ \\
\hline 2MASS02394528-3431581 & Fornax 26 & 15.941 & 14.989 & 14.667 & & - & not observed \\
\hline 2MASS02410355-3448053 & Fornax 27 & 14.441 & 13.365 & 12.694 & -4.59 & $\mathrm{C}$ & 1.53 absent; $(3,4,7)$ \\
\hline 2MASS02402039-3437345 & Fornax 28 & 15.930 & 14.875 & 14.649 & & - & not observed \\
\hline 2MASS02402554-3441585 & Fornax29 & 15.784 & 14.926 & 14.439 & & - & not observed \\
\hline 2MASS02410145-3437154 & Fornax30 & 15.803 & 14.737 & 14.401 & & - & not observed \\
\hline 2MASS02380618-3431194 & Fornax31 & 16.052 & 14.483 & 13.315 & -4.03 & $\mathrm{C}$ & 1.53 medium \\
\hline 2MASS02385700-3446340 & Fornax 32 & 14.789 & 13.664 & 13.076 & -4.21 & $\mathrm{C}$ & 1.53 absent; (7) \\
\hline 2MASS02392952-3449431 & Fornax33 & 15.905 & 15.144 & 14.644 & & - & not observed \\
\hline \multirow[t]{5}{*}{ 2MASS02385056-3440319 } & Fornax34 & 16.106 & 14.525 & 12.879 & -4.70 & $\mathrm{C}$ & 1.53 extreme; $(5,6,7)$ \\
\hline & Fornax-S66 & 14.768 & 13.901 & 13.642 & & M2S & \# 66 from Stetson et al. (1998) \\
\hline & Fornax-S71 & - & - & - & & K5 & \# 71 from Stetson et al. (1998) \\
\hline & Fornax-S99 & - & - & - & & $\mathrm{S}$ & \# 99 from Stetson et al. (1998) \\
\hline & Fornax-S116 & 15.004 & 14.041 & 14.028 & & $\mathrm{SC}$ & \# 116 from Stetson et al. (1998) \\
\hline 2MASS01002467-3352012 & Scl1 & 15.024 & 14.280 & 13.791 & -2.92 & non stellar & 2MASX source \\
\hline 2MASS01005598-3352344 & Scl2 & 15.977 & 15.290 & 14.610 & -1.97 & K2-K4 & $H$ band curvature odd (background?) \\
\hline 2MASS00590481-3343297 & $\mathrm{Scl} 3$ & 16.118 & 15.283 & 14.688 & & - & not a point source, no spectrum taken \\
\hline 2MASS01004469-3327134 & Scl4 & 15.367 & 14.643 & 14.133 & -2.57 & non stellar & 2MASX, 2dFGRS S503Z077 $(z=0.11)$ \\
\hline 2MASS00595925-3337091 & Scl5 & 15.963 & 15.284 & 14.565 & & - & not observed \\
\hline 2MASS00595367-3338308 & Scl6 & 14.846 & 13.144 & 11.603 & -4.93 & $\mathrm{C}$ & 1.53 strong; (4) \\
\hline 2MASS00593953-3332545 & Scl7 & 15.264 & 14.501 & 14.018 & -2.67 & non stellar & 2MASX source \\
\hline \multirow[t]{5}{*}{ 2MASS01005213-3343136 } & Scl8 & 15.845 & 15.171 & 14.543 & -2.10 & non stellar & 2MASX source \\
\hline & Scl-Az1-C & 14.713 & 14.040 & 13.871 & & $\mathrm{C}$ & ALW1 from Azzopardi et al. (1985) \\
\hline & Scl-Az3-C & - & - & - & & $\mathrm{C}$ & ALW3 from Azzopardi et al. (1985) \\
\hline & Scl-scms-209 & 13.628 & 13.080 & 12.823 & & M4 & from Schweitzer et al. (1995) \\
\hline & Scl-scms-1448 & 15.167 & 14.497 & 14.340 & & M3 & from Schweitzer et al. (1995) \\
\hline 2MASS19445931-1446010 & N6822-1 & 16.222 & 15.148 & 14.737 & & - & not observed \\
\hline 2MASS19444196-1440072 & N6822-2 & 15.889 & 14.766 & 14.417 & -5.93 & K4-M & \\
\hline 2MASS19450019-1446040 & N6822-3 & 15.189 & 14.234 & 13.760 & & - & not observed \\
\hline 2MASS19443399-1446237 & N6822-4 & 15.593 & 14.519 & 14.231 & -6.22 & M & $\mathrm{H}_{2} \mathrm{O}$ absorption \\
\hline 2MASS19445849-1448037 & N6822-5 & 16.142 & 15.314 & 14.785 & & - & double star, no spectrum taken \\
\hline 2MASS19444529-1451252 & N6822-6 & 16.083 & 15.059 & 14.553 & & - & not observed \\
\hline 2MASS19445689-1442381 & N6822-7 & 16.064 & 15.104 & 14.610 & -5.76 & M & $\mathrm{H}_{2} \mathrm{O}$ absorption \\
\hline 2MASS19444914-1449051 & N6822-8 & 16.042 & 14.913 & 14.633 & -5.78 & K4-M & \\
\hline 2MASS19444900-1444334 & N6822-9 & 15.230 & 14.204 & 13.738 & & - & not observed \\
\hline \multirow[t]{3}{*}{ 2MASS19452775-1453299 } & N6822-10 & 15.383 & 14.415 & 14.022 & -6.43 & M & $\mathrm{H}_{2} \mathrm{O}$ absorption \\
\hline & LHS 517 & 6.510 & 5.899 & 5.594 & & M3.5 dwarf & \\
\hline & LHS 3788B & 9.459 & 8.840 & 8.531 & & M5 dwarf & \\
\hline
\end{tabular}

1 = Demers \& Kunkel (1979); 2 = Westerlund et al. (1987); 3 = Bersier \& Wood (2002); 4 = Mauron et al. (2004); 5 = Matsuura et al. (2007);

$6=$ Lagadec et al. (2008); 7 = Whitelock et al. (2009). 

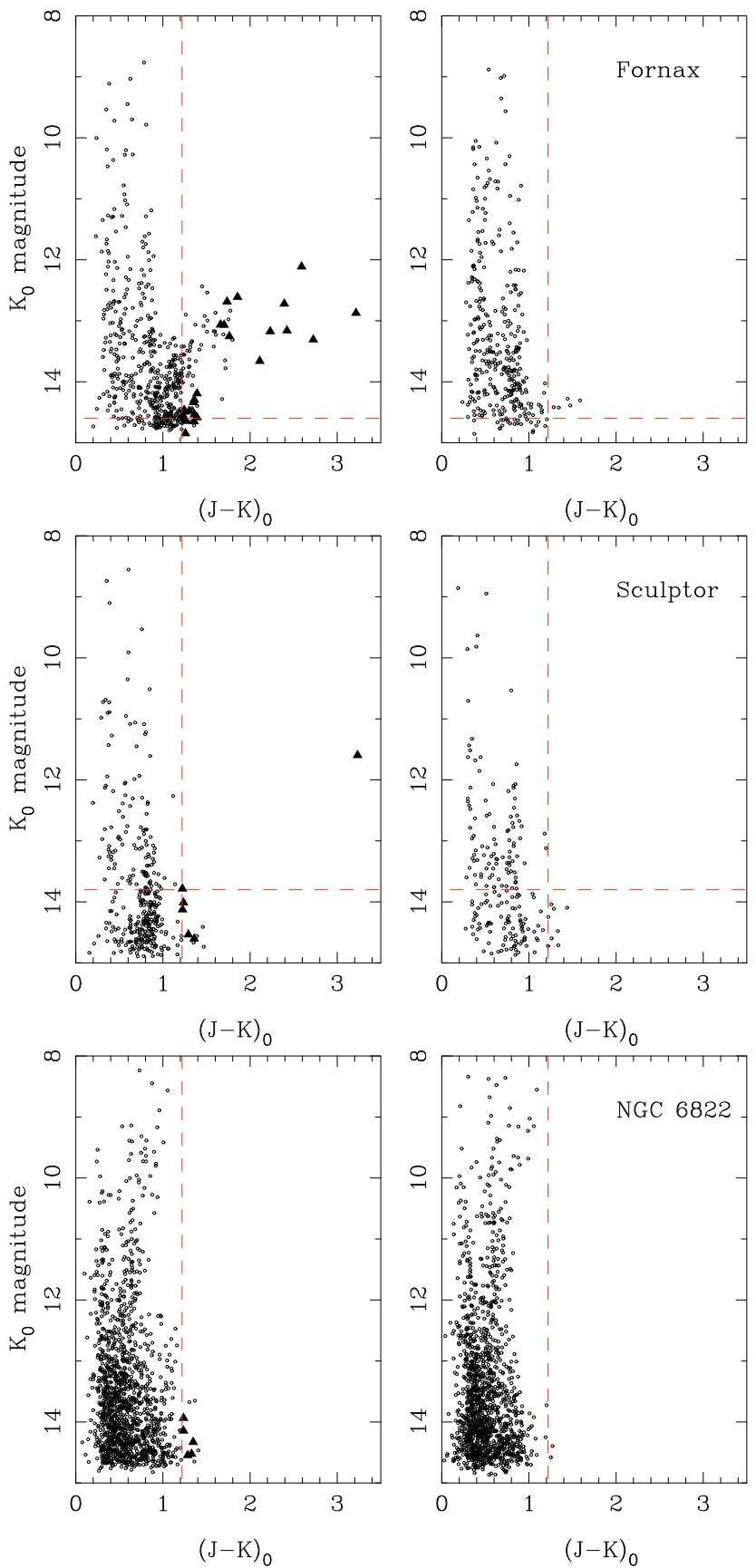

Fig. 1. Colour-Magnitude diagram for Fornax, Sculptor and NGC 6822 (left) and their surroundings (right). Stars for which a spectrum was obtained are marked by the filled triangles. The dashed vertical line indicates the lower limit of $(J-K)_{0}=1.22$ used in selecting late-M and C-stars. The dashed horizontal line indicates the tip of the RGB. For NGC 6822 it is located at $K_{0}=17.0$, beyond the limit of the plot (see text).

The on-chip integration time was $120 \mathrm{~s}$ for the science targets and $5 \mathrm{~s}$ or less for the telluric standard star observations. A standard nodding technique was used with a throw of $20^{\prime \prime}$. To build up signal-to-noise 1-6 nod cycles were taken depending on the brightness of the target. A slit of $1^{\prime \prime}$ was used. A telluric standard was observed immediately after each science target. It was taken from the Hipparcos database in the direction of the science target and was preferentially a hot star (B8 or earlier spectral type), or otherwise a G2 solar analog.
The basic data reduction was done using the ESO offered ISAAC data reduction pipeline (version 5.4.2) for flat-fielding, wavelength calibration (using the sky lines), correction for the slit curvature and the star trace, combination of the nod positions and cycles and the detection and extraction of the final spectrum, that was written to an ascii file.

The task of removing the telluric features was done in a separate Fortran code. The basic idea is to divide the observed spectrum of the science target by that of the telluric standard and multiply the result by the intrinsic spectrum of the standard.

A high-resolution near-infrared spectrum of the Sun was taken from Castelli et al. (1997), and those for early-type stars were kindly calculated and made available by Peter Hauschildt. An approximate absolute flux level was achieved by scaling the theoretical spectrum for the telluric standards to a flux level based on the 2MASS $J$ and $H$ magnitudes, but no corrections for slit losses were made.

The high-resolution spectra of the standard stars were convolved to lower resolution matching the ISAAC observations. To further judge the quality of the removal of the telluric feature we convolved the high-resolution telluric spectrum available at the ESO website ${ }^{2}$ to the resolution of the observations and compared it to the final science spectrum in particular in parts of the spectrum with strong telluric features.

The final spectra are shown in Fig. 6, which is available in its entirety in electronic form via http: //WwW . aanda. org. The ordinate is in $\mathrm{W} / \mathrm{m}^{2} / \mu \mathrm{m}$ and is on an approximate absolute level.

\section{Description of the spectra and classification}

\subsection{Classification criteria}

At the resolution of our observations, the main features seen in the spectra of luminous red stars are molecular bands, although line and line blends due to neutral atoms are also present. Spectra useful for rapid band identifications and classification can be found among others in Lançon et al. (1999) or Lançon \& Wood (2000, LW2000 hereafter), in Gautschy-Loidl et al. (2004) for carbon stars, in Joyce et al. (1998) for S stars, in McLean et al. (2003) or Cushing et al. (2005) for M dwarfs and brown dwarfs. In the case of O-rich stars, the sensitivity of the spectra to metallicity is larger than for carbon stars. Our comparison sample therefore includes six near-IR spectra of Population II giants (type G2 to K3.5), of which five were kindly provided by Vacca (Fig. 2). Finally, we may exploit the theoretical and empirical spectra of red supergiants analysed in Lançon et al. (2007), and the SpeX/IRTF library of near-IR spectra of Milky Way stars of all types (Vacca et al. and Rayner et al., in preparation) ${ }^{3}$.

The features most useful for the classification of our spectra of O-rich giants are the molecular bands and line blends in the $\mathrm{H}$-band (mostly $\mathrm{CO}$ and $\mathrm{OH}$, but also metal lines; see e.g. Origlia et al. 1993, Frémaux et al. 2006), and the curvature of the spectrum across the $H$-band (first used for classification purposes by Terndrup et al. 1991). As seen in Fig. 2, the (negative) curvature of the $H$-band increases with decreasing temperature between types $\mathrm{G} 2$ and $\mathrm{K} 4$, as a result of the combined effects of the varying importance of $\mathrm{H}^{-}$opacity (which has a minimum around $1.5 \mu \mathrm{m}$ ) and of $\mathrm{CN}$ absorption (which has a broad band on the short wavelength side of the H-window). The $\mathrm{CN}$ bandhead at $1.1 \mu \mathrm{m}$, which had been a motivation for our $J$ band observations, turns out to be difficult to use because it lies

\footnotetext{
2 http://www . eso.org/sci/facilities/paranal/

instruments/isaac/tools/spectroscopic_standards.html

3 http://irtfweb. ifa.hawaii .edu/ spex/
} 

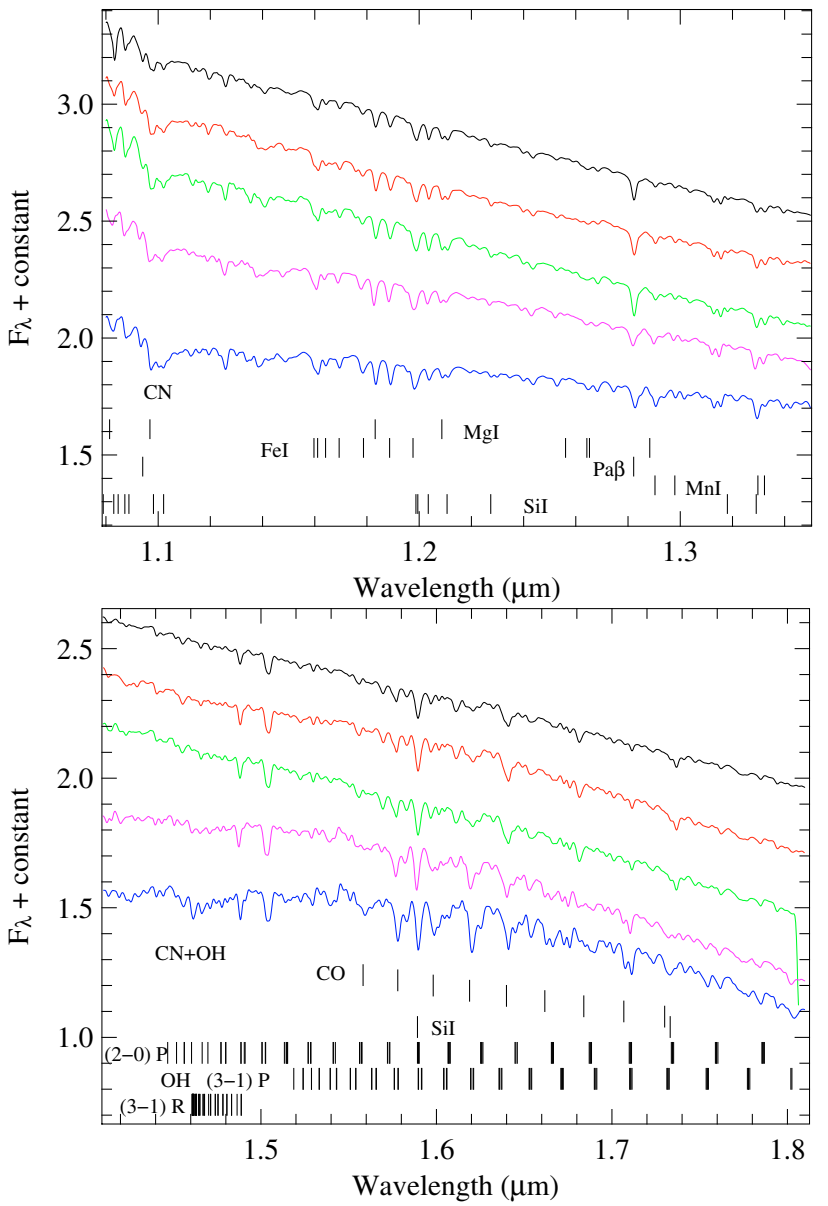

Fig. 2. Spectra of metal-poor giants (Vacca, priv. comm.). Estimated metallicities from the literature are scattered between $[\mathrm{Fe} / \mathrm{H}]=-0.4$ to -1 . Spectral types from top to bottom are G3 (HD 88639), G8 (HD 135722), G8.5 (HD 16139), K2 (HD 2901) and K3.5 (HD 99998). $\mathrm{OH}$ line identifications are taken from Maillard et al. (1976).

at the edge of the detected spectral range and has poor signalto-noise ratios. $\mathrm{H}_{2} \mathrm{O}$, when present, is expected to curve the $\mathrm{H}$ window spectrum even more because of absorption bands on either side. It also displays a sharp bandhead at $1.34 \mu \mathrm{m} . \mathrm{H}_{2} \mathrm{O}$ in red giants is interpreted as a signature of Mira-type long period variability (LW2000, Tej et al. 2003). It is also present in low mass dwarfs, which in our case would be foreground contaminants. $\mathrm{VO}$ and $\mathrm{TiO}$ features will show in the $J$-band spectra of stars very low effective temperatures (late $\mathrm{M}$ type). Evolutionary tracks do not predict such temperatures at low metallicity, but long period variability can extend atmospheres enough to produce at least the VO band at $1.04 \mu \mathrm{m}$ (see e.g. the population II Mira-type variable S Carinae, LW2000).

Carbon stars are characterised by strong bands of the red system of cyanogen $(\Delta v=0$ bandhead of $\mathrm{CN}$ at $1.09 \mu \mathrm{m}, \Delta v=-1$ band responsible for structure throughout the $H$-window, e.g. the $(1,2)$ band at $1.45 \mu \mathrm{m})$ and by the Ballik-Ramsey system of $\mathrm{C}_{2}$ ( $\operatorname{sharp} \Delta v=0$ bandhead at $1.77 \mu \mathrm{m}$, and $\Delta v=+2$ bands at 1.17 , $1.20,1.23 \mu \mathrm{m}$ ). Extreme carbon stars (in a sense that will be further discussed later) display a band around $1.53 \mu \mathrm{m}$, for which the carrying molecule has been a matter of debate (e.g. LW2000, Joyce 1998 and references therein) and that is currently assigned mainly to $\mathrm{C}_{2} \mathrm{H}_{2}$ (Gautschy-Loidl et al. 2004). Carbon stars also show the second overtone ro-vibrational $\mathrm{CO}$ absorption band series longwards of $1.56 \mu \mathrm{m}$. This series has a particularly regular appearance in $\mathrm{S}$ and some $\mathrm{S} / \mathrm{C}$ stars (see Fornax-S99 and Fornax-S116 in the present paper and the S/C star BH Crux from LW2000).

\subsection{Background galaxy contaminants}

A few of our initial targets appeared clearly extended on the acquisition image and no spectrum was taken as indicated in Table 1.

Six spectra (4 in Sculptor, 2 in Fornax) clearly show a positive curvature in the $H$-band. One more $(\mathrm{Scl} 2)$ displays some similarity with the latter, but the curvature is less pronounced. At the data reduction stage the acquisition image of all targets were inspected and the FWHM of the target and, when available, other stellar sources in the field, were measured. The evolution of the FWHM of the carbon stars and the spectral reference sources through the night gave an indication of the evolution of the image quality, and this showed that the 6 first odd-spectrum sources are extended. One was found listed in the $2 \mathrm{dF}$ Galaxy Redshift Survey with a redshift of 0.11 : the dip seen in the $H$-band spectra is due to the combined effects of the $\mathrm{H}^{-}$opacity minimum near rest wavelength $1.6 \mu \mathrm{m}$, and molecular bands (mainly $\mathrm{CN}$ ) and the Brackett jump near rest wavelength $1.46 \mu \mathrm{m}$. All 6 objects are likely low-redshift galaxies. Scl2 might be one as well, although it is not resolved (see Morris et al. 2007).

\subsection{Fornax data}

The initial search of 2MASS objects in the direction of Fornax fulfilling the criteria mentioned in Sect. 2 resulted in a list of 155 known late-type stars, all from the work by Stetson et al. (1998) and references therein. Out of the ones with a known spectral type, 19 have spectra type M (or MS), $7 \mathrm{~S}$ (or SC), and $39 \mathrm{C}$ Out of the 34 new AGB stars candidates in Fornax, 25 have been observed. We provide classification for 22 stars in Table 1. Of the 3 remaining targets, two are likely low-redshift galaxies, and the third has such a peculiar, noisy $H$-band spectrum that we do not classify it. We do not include them in our discussion.

Out of the 22 useful program stars, 11 are O-rich giants and 12 are carbon stars. Little was previously known about these objects. Some of the stars we now classify as C-stars had been previously identified as very red objects. Since the submission of the observing proposal, one Fornax star has been identified as a C-star by Mauron et al. (2004) based on optical spectra in a survey for C-stars in the Galactic Halo. Matsuura et al. (2007) obtained Spitzer IRS spectra for 3 objects, and Lagadec et al. (2008) obtained mid-IR images for 2 objects. Recently, Whitelock et al. (2009) present the result of a near-infrared survey of the inner $42 \times 42$ arcmin. Several of the C-stars in our sample are in their lists of variable stars (see Table 1). None of our targets is among their selection of non-variable upper AGB candidates.

There is a clear distinction between the $\mathrm{C}$ - and the non-C-stars we observed in Fornax. All stars redder than $(J-$ $K)_{0}=1.63$ are C-stars, all stars bluer than $(J-K)_{0}=1.40$ are Orich. A similar distinction exists in the bolometric magnitudes: all O-rich stars in our sample are fainter than $M_{\text {bol }}=-3.42$, while C-stars are brighter than -3.55 , reaching -5.2 for the reddest one.

What is interesting is the apparent difference between the ratio of C- to O-rich stars found by Stetson et al., 39/26 = 1.5, and in this study, $12 / 11=1.1$. The true ratio is probably even 
smaller as the $(J-K)$ colours of the stars not observed indicate they should be $\mathrm{O}$-rich, in which case the $\mathrm{C} / \mathrm{O}$ ratio drops to $12 / 19=0.63( \pm 0.22$ if one considers Poisson errors $)$. Many of the stars in Stetson et al. do not have a spectral classification and it appears that these are preferentially O-rich stars. To investigate this, we have divided the Stetson et al. sample in 4 bins of $(B-R)$ colour and counted the number of known $\mathrm{C}, \mathrm{S} / \mathrm{SC}$ and $\mathrm{M} / \mathrm{MS}$ stars per colour bin. The non-classified stars were then classified on pro rata basis. The estimated number of $\mathrm{C}, \mathrm{S} / \mathrm{SC}$ and $\mathrm{M} / \mathrm{MS}$ in the Stetson et al. sample of red stars is, respectively, 78, 23 and 59, corresponding to a $\mathrm{C} / \mathrm{O}$ ratio of $0.95( \pm 0.14$ if one considers Poisson errors). This ratio does not depend on any reasonable choice of the colour bins. The conclusion is that the ratio of $\mathrm{C}$ to non- $\mathrm{C}$ stars in the optical and 2MASS sample are the same within the errors.

In order to provide a tentative spectral type for the O-rich giants, we have compared them individually with the reference spectra available. The spectral features in the data, such as the $\mathrm{CO}$ bands, are weak. Poor matches are therefore obtained with high luminosity stars and with most of the spectra of LW2000. On the contrary, good matches are obtained with the spectra of Fig. 2. We caution that spectral types were originally defined based on optical spectra of solar neighbourhood stars: little is known about the systematics that affect the relation between spectral type and near-IR properties for cool, metal poor stars (Gray 2009). As an example, the K3.5 giant of our reference sample, with $[\mathrm{Fe} / \mathrm{H}] \sim-1$, is about $0.2 \mathrm{mag}$ redder in $(J-K)$ than solar neighbourhood stars of the same spectral type (LW2000 and IRTF spectral library). It has very similar near-IR colours and features to a solar neighbourhood star of type M1III. Clearly, the spectral types assigned here are preliminary and metallicity-related systematics would require dedicated studies with large samples of optical and near-IR spectra.

In the process of comparison, the $J$ - and $H$-band ISAAC spectra were rescaled separately to match the flux levels of the reference spectra. The scaling factors never differed by more than $20 \%$, which indicates that slit losses did not vary too severely between our observations. An illustration is given in Fig. 3. The curvature of the $H$-band spectrum together with the slope within the $J$-band are the most discriminant features in this limited set of comparisons. At the signal-to-noise ratio of the data, individual narrow spectral features provide no significant additional discrimination. We also explored to what extent reddening corrections could affect the classification (using the extinction law of Cardelli et al. 1989). In about half the cases, equally good fits were obtained with the data dereddened by up to $A_{\mathrm{V}} \sim 1$, but with slightly earlier spectral types. The ranges of spectral types given in Table 1 are all inclusive.

Assigned spectral types extend from G8 to K3.5, K3.5 being the limit of our comparison sample of metal poor giants. We identify no obvious candidate for a much later spectral type than $\mathrm{K} 4$. The absence of $\mathrm{H}_{2} \mathrm{O}$ features is in agreement with this statement. It argues against contamination by foreground $\mathrm{M}$ dwarf stars. In addition, it suggests the absence of large amplitude variables in the O-rich sample.

\subsection{Sculptor data}

The initial search of 2MASS objects in the direction of Sculptor fulfilling the criteria mentioned in Sect. 2 resulted in a list of 14 knows AGB stars: 8 C-stars from Azzopardi et al. (1985), and 3 C- and 3 M-stars from Schweitzer et al. (1995).

Of the 8 candidate AGB stars, 6 targets have been observed in the Sculptor field, and four are selection errors (low-redshift

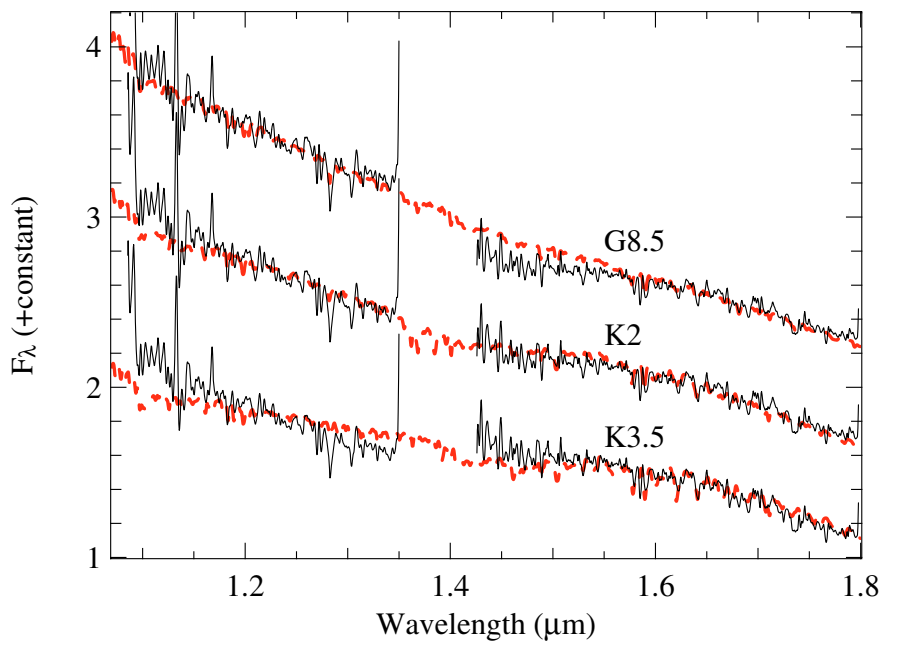

Fig. 3. Illustration of the comparison between a Fornax star spectrum and the spectra of metal poor giants from Fig. 2. The case of Fornax 22 is shown (solid black) with three comparison spectra (dashed red). The flux zero point holds for the bottom spectra; a constant has been added to the other sets of spectra for display.

galaxies with a characteristic rise in the $H$-band spectrum; one has a known redshift of 0.11 ). One is either an O-rich giant or an unresolved background galaxy. One is a carbon star. This is a remarkable object as it is redder than all $12 \mathrm{C}$-stars detected in Fornax and the second brightest object of our whole sample in bolometric magnitude. Its spectrum is also exceptional, as it displays the strongest $1.53 \mu \mathrm{m}$ feature of all our C-star observations (see Sect. 6.2).

\subsection{NGC 6822 data}

The initial search of 2MASS objects in the direction of NGC 6822 fulfilling the criteria mentioned in Sect. 2 resulted in a list of 130 known AGB stars. With the exception of one S-star (Aaronson et al. 1985) and one M-star (Elias \& Frogel 1985), they all come from the C-star survey presented by Letarte et al. (2002) which contains a total of 904 C-stars.

Of the 10 new candidate AGB stars, five were observed in NGC 6822 and all are O-rich, although hundreds of C-stars have been identified earlier in this dwarf galaxy (Letarte et al. 2002; Demers et al. 2006). They are also very bright: with bolometric magnitudes smaller than -5.76 , they lie near or above the bright end of the C-star luminosity function for NGC 6822 presented by Groenewegen (2007), based on the $R, I$ photometry of Letarte et al. (2002).

Three of the five stars are likely Mira-like long period variables as they display a sharp bandhead at $1.34 \mu \mathrm{m}$ due to $\mathrm{H}_{2} \mathrm{O}$ (numbers 4, 7 and to a slightly lesser extent 10). We cannot exclude that the other two are also of this type, since Mira-like stars do not always show $\mathrm{H}_{2} \mathrm{O}$ (e.g. LW2000). When compared with the O-rich stars observed in Sculptor and in Fornax, or with the reference low metallicity giants of Fig. 2, the O-rich stars in NGC 6822 display stronger features of $\mathrm{CO}$ and $\mathrm{OH}$ across the $H$-band. The strength of these features is known to increase with luminosity. Considering the bolometric magnitudes of our targets, this result is not surprising.

The dereddened $(J-K)$ colours are in the range 1.23 to 1.40 , when a mean foreground extinction is applied. We note that foreground extinction is variable across the field of NGC 6822 with known differences of about $0.4 \mathrm{mag}$ in $A_{\mathrm{V}}$ (Wyder 2003). For 

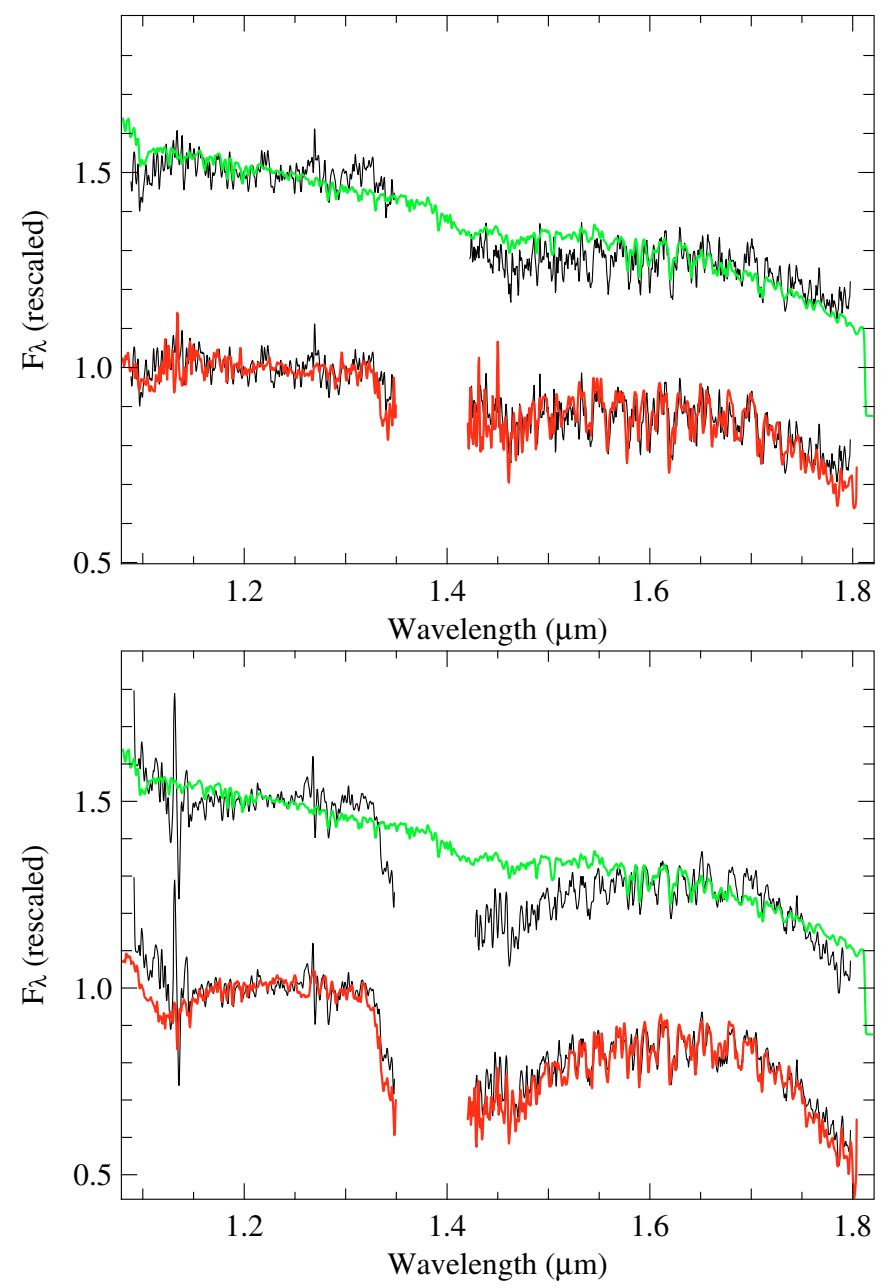

Fig. 4. Upper panel: comparison of NGC 6822-8 (black lines) with the coolest spectrum of Fig. 2 (top) and with the May 1996 spectrum of the Milky Way long period variable S Phe (bottom). Lower panel: same as above for NGC 6822-4, but with the April 1995 spectrum of U Crt as a Milky Way template. In both panels, 0.5 flux units have been added to the upper spectra for display.

the five observed stars, we find a better match to the coolest metal-poor spectrum of Fig. 2 (type K3.5) with an $A_{\mathrm{V}}$ of 0.5 to 2. This suggests circumstellar extinction, or a spectral type later than K4. Considering how much the near-IR energy distribution of a Mira variable can change, it is difficult to find the perfect template, and thus to argue one way or the other. We performed comparisons with the Milky Way Mira type spectra that had similar $\mathrm{CO}$ band strengths and $H$-band curvatures (LW2000), and found no need for extra extinction with those templates. Of course, these Milky Way stars could be reddened themselves, and they are likely to be more metal rich than those in NGC 6822. Two examples are given in Fig. 4.

\section{Discussion}

\subsection{Evolutionary considerations}

Because our samples are not complete, they can not be used to put strict constraints on the star formation histories of galaxies. Therefore, we restrict this section to a brief verification of consistency with theoretical predictions, using history determinations in the literature. For the effects of metallicity on the location of the AGB in colour-magnitude diagrams and the formation of carbon stars, we have used the work of Mouhcine (2002), Mouhcine \& Lançon (2003), Marigo \& Girardi (2007) and Marigo et al. (2008).

\section{- Fornax}

As summarised by Coleman et al. (2005), based in particular on the work of Stetson et al. (1998), Buonanno et al. (1999), Saviane et al. (2000), Tolstoy et al. (2003) and Pont et al. (2004), star formation has occurred in Fornax over long timescales. An old low metallicity giant branch coexists with an important young population, born over the last few Gyr with metallicities centered on $[\mathrm{Fe} / \mathrm{H}]=-1$ dex. Many carbon stars testify of this intermediate age population (Aaronson \& Mould 1980; Azzopardi et al. 1999). The youngest stars may be as young as a few $100 \mathrm{Myr}$, and as metal rich as $[\mathrm{Fe} / \mathrm{H}]=-0.4$ dex.

The C-stars in our sample have bolometric magnitudes between -3.56 and -5.12 . Such luminosities are consistent with model predictions. The luminosity of the brightest C-star translates into an age younger than $1 \mathrm{Gyr}$. The faintest $\mathrm{C}$-star is compatible with the quiescent $\mathrm{H}$-burning luminosity along the TP-AGB only for $[\mathrm{Fe} / \mathrm{H}]<-1$ (Marigo \& Girardi 2007). Alternatively, this star could be evolving through one of the luminosity dips that follow helium flashes, or be the result of binary evolution.

We find that the O-rich stars in our sample are systematically fainter than the $\mathrm{C}$-rich stars, with bolometric magnitudes between -2.9 and -3.4 . These luminosities lie just below the transition between the early-AGB and the thermally pulsing AGB. Early spectral types and the lack of evidence for large amplitude pulsation are therefore not surprising.

\section{- Sculptor}

The fraction of intermediate age stars is smaller in Sculptor than in Fornax. Sculptor is believed to have formed most of its stars more than $10 \mathrm{Gyr}$ ago, although star formation then continued at a lower level until it stopped several Gyr ago (Tolstoy et al. 2003). The metallicities of the oldest stars are clustered around $[\mathrm{Fe} / \mathrm{H}]=-2$ dex (Tolstoy et al. 2004; Clementini et al. 2005); a more centrally concentrated and kinematically distinct stellar population has a typical $[\mathrm{Fe} / \mathrm{H}]$ of -1.4 dex (Tolstoy et al. 2004; Babusiaux et al. 2005).

The single O-rich star in the Sculptor sample is relatively faint (bolometric magnitude of -2). This star is likely to belong to the early-AGB. The one C-rich star we observed, on the contrary, is bright $\left(M_{\text {bol }} \simeq-4.9\right)$. Stellar evolution predicts that such a luminosity is reached in the quiescent H-burning phases of the TP-AGB only for progenitor masses near $2 M_{\odot}$ or higher, which translates into ages of $\sim 2$ Gyr or less. This star might be experiencing a helium flash, or it may have followed a non-standard evolutionary path (a few isolated examples of old C-rich TP-AGB isochrones are shown in the low metallicity grids of Marigo et al. 2008). Because it is a known variable, it is unlikely to be a dwarf carbon star in the foreground (Mauron et al. 2004).

\section{- NGC 6822}

NGC 6822 is a Magellanic irregular galaxy with a bar, an ellipsoidal component known to host carbon stars, and an extended HI polar ring apparently devoid of old and intermediate age stars (Demers et al. 2006; de Blok et al. 2006). The galaxy is thought to have formed stars over a large fraction of the age of the universe, with possible fluctuations such as the differences between the star formation rates seen in the bar and around it over the past few hundred million years (Gallart et al. 1996 a,b; Wyder 2003). The metallicities of the younger stars are 2 or 3 times below solar (e.g. Venn et al. 2001). 
The stars observed with ISAAC are bright. Evolutionary tracks for the thermally pulsating AGB reach those luminosities for initial masses above $3.5 M_{\odot}$, or ages younger than about $500 \mathrm{Myr}$. Stars in this range spend no or very little time as C-stars before loosing their envelope, because of combined effects of hot-bottom burning (which burns $\mathrm{C}$ into $\mathrm{N}$ at the base of the convective envelope, when sufficiently hot) and mass loss (which radically reduces TP-AGB lifetimes when luminosity is high). The observed O-rich atmospheres are fully consistent with the estimated luminosities.

\subsection{The $1.53 \mu \mathrm{m}$ feature of carbon stars and mass loss}

The observations of stars with 2MASS colours $(J-K)>1.22$ has resulted in the confirmation of a dozen carbon stars in Fornax and Sculptor. The presence of the sharp $\mathrm{C}_{2}$ bandhead at $1.77 \mu \mathrm{m}$ was adopted as a sufficient condition for a classification among carbon stars. Most of the carbon stars also display the characteristic $\mathrm{C}_{2}$ band that spreads between $1.15 \mu \mathrm{m}$ and $1.3 \mu \mathrm{m}$, as well as the $\mathrm{CN}$ absorption bandhead at $1.1 \mu \mathrm{m}$.

The most surprising result of the present work is the frequent detection of the so-called $1.53 \mu \mathrm{m}$-feature (Goebel et al. 1981; Joyce 1998) in the sample of carbon stars. This band was previously noticed in a few low temperature carbon stars of the Milky Way (Goebel et al. 1981; Joyce 1998). The strength of the band was shown to depend on pulsation phase in C-rich Mira variables such as V Cyg or R Lep (Joyce 1998, LW2000). After some debate, it is now considered likely that the main carrier of this feature is the $\mathrm{C}_{2} \mathrm{H}_{2}$ molecule, although the predicted shape of the band in dynamical models of cool carbon stars does not quite match the observed shapes as yet and HCN may contribute (Gautschy-Loidl et al. 2004). The $1.53 \mu \mathrm{m}$ band would then be an overtone of one of the bands contributing to the much stronger, and more common, $3.1 \mu \mathrm{m}$ feature of carbon stars (see e.g. van Loon et al. 2006, 2008, for a sample in the LMC).

Although incomplete, our sample may help characterise when the $1.53 \mu \mathrm{m}$ feature appears. The upper left panel of Fig. 5 shows the passbands used to measure the strengths of various bands. When possible, two "continuum" regions 4 were selected on either side of the region of interest, in order to reduce sensitivity to extinction or to broader overlapping absorption features. This was not possible for $\mathrm{C}_{2}(1.77 \mu \mathrm{m})$, and large uncertainties near the short wavelength edge of the $J$-band spectra made us abandon the measurement of the $\mathrm{CN}$ bandhead $(1.10 \mu \mathrm{m})$. One or several regions were used for the measurement of the flux within the molecular bands of interest. The strength of any absorption band $X$ is measured as

$I(X)=2.5 \log \left(F_{\mathrm{c}}(X) / F_{\mathrm{b}}(X)\right)$

where $F_{\mathrm{c}}(X)$ (resp. $\left.F_{\mathrm{b}}(X)\right)$ is the mean energy density received in the wavelength bins of the "continuum" region(s) (resp. in the absorbed regions).

Figure 5 shows the measurements obtained. The following comments can be made:

- the programme stars are offset from the S and C stars used for reference, because these are intrinsically bluer;

- the strengths of the $\mathrm{C}_{2}$ features measured in the $J$-band and in the $H$-band correlate well;

- the $1.53 \mu \mathrm{m}$ feature is found only when the $\mathrm{C}_{2}$ features are relatively weak. This is consistent with a picture in which

\footnotetext{
4 The real continuum is nowhere accessible in carbon stars with observations at the resolution of our data.
}

carbon $\mathrm{C}_{2} \mathrm{H}_{2}$ formation happens at the expense of $\mathrm{C}_{2}$ in the outer atmosphere, when layers of sufficiently cool temperature and sufficiently high density exist (Gautschy-Loidl et al. 2004; Jorgensen priv. comm.);

- the $1.53 \mu \mathrm{m}$ features shows a positive correlation with $J-K$, while the strength of the $\mathrm{C}_{2}$ features in our sample shows the opposite trend. The extinction vectors show that a few magnitudes of (optical) circumstellar extinction will not modify the trends. Incompleteness is more of a concern. The trends with $J-K$ are consistent with the picture above, if $J-K$ can be taken as a first order indication of the temperatures present in the emitting layers of the atmosphere.

The dynamical model atmospheres of Gautschy-Loidl et al. (2004) show that the $1.53 \mu \mathrm{m}$ band appears in their coolest models $\left(T_{\text {eff }}<2800 \mathrm{~K}\right)$, which start developing a wind, near minimum light. The band strength shown in Gautschy-Loidl et al. is much less than observed in some of the extreme cases observed here, but this particular model has a mass-loss rate of $6.6 \times 10^{-7} M_{\odot} \mathrm{yr}^{-1}$. The picture that is emerging based on the available observational data and theoretical model atmospheres is that the $1.53 \mu \mathrm{m}$ band is a function of phase in the pulsation cycle, strongest at minimum light, and that its strength increases with mass-loss.

We have attempted to test this further by cross-identifying our sample with the photometric multi-epoch survey of Fornax by Whitelock et al. (2009) and with Spitzer-IRS data, also on Fornax, presented with derived mass loss estimates by Matsuura et al. (2007). All the objects Whitelock et al. marked as showing low amplitude semi-regular or irregular variations display no or a weak $1.53 \mu \mathrm{m}$ feature. Unfortunately, very small number statistics prevent of from identifying any other trends. The diversity in behaviours suggests a large dispersion in any such trend, as a natural result of the complexity induced by pulsation.

\section{Conclusions}

Based on the 2MASS point source catalog, we have selected candidate AGB stars in Local Group dwarf galaxies by requiring a large intrinsic $(J-K)$ colour index as well as a AGB-like bolometric luminosity (Groenewegen 2006). The candidates in Fornax, NGC 6822 and Sculptor not previously confirmed spectroscopically are listed in Table 1 , and $J$ and $H$-band spectra were obtained for about two thirds of the candidates. These spectra allow us to identify carbon stars and to estimate the spectral types of oxygen-rich AGB stars, as well as to recognise and reject contaminating background galaxies.

In Fornax, 12 of the 22 stars observed are carbon stars, and this can be compared to the list of 104 carbon stars identified previously (Azzopardi et al. 1999). In NGC 6822 on the other hand, all 5 stars observed are O-rich and not very red $(J-K \sim 1.4)$. This is likely a selection effect. Because of distance the $J$ and $K$ magnitudes are close to the detection limit. Any obscured Cor O-rich star would likely not be detected in $J$ and hence not be in the sample of candidate AGB stars. As mentioned in Sect. 5.5 the luminosity of the O-rich stars is close to or brighter than the bright end of the C-star LF as determined from the Letarte et al. sample. It would be interesting to observe these stars at high resolution in the optical and look for signatures of Hot Bottom Burning. Our work did not add any new C-stars, but to exclude the presence of mass-losing O- and C-stars would require a survey to fainter magnitudes. In Sculptor, our initial sample is strongly contaminated by background galaxies. The only carbon star we find is the extremely red object also mentioned 

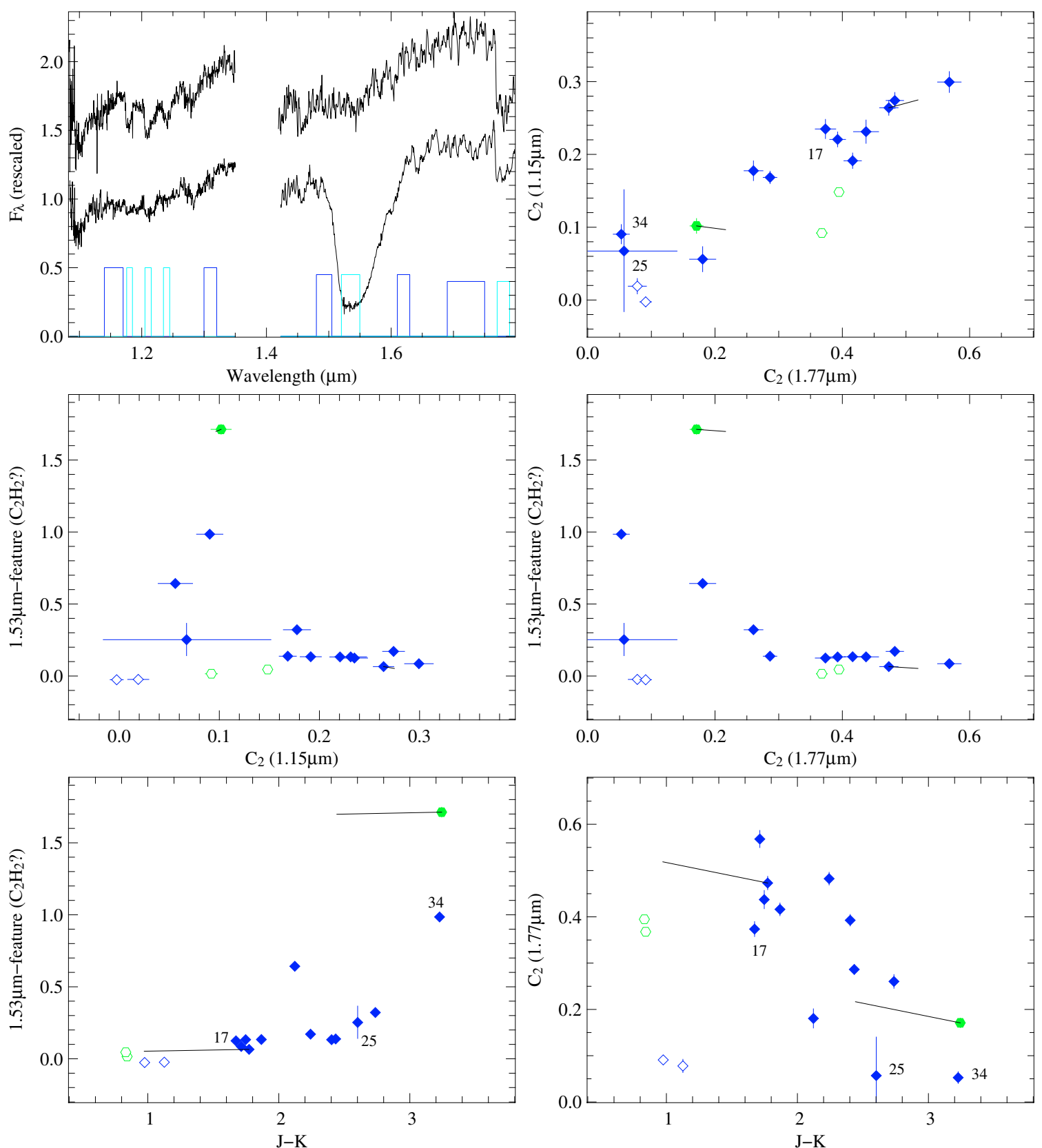

Fig. 5. Upper left: spectral index definition passbands. The (arbitrary) passband heights indicate passbands used for measuring $\mathrm{C}_{2}$ around $1.15 \mu \mathrm{m}$ (highest), the $1.53 \mu \mathrm{m}$ feature (medium), and $\mathrm{C}_{2}$ at $1.77 \mu \mathrm{m}$ (lowest). "Continuum" passbands are marked in blue, "feature" passbands in cyan. The spectra shown are those of Fornax 21 (top) and Sculptor 6. Other panels: index measurements on the carbon star spectra. Stars in Fornax are shown as dark blue diamonds, stars in Sculptor as green hexagons. Open symbols are reference stars, solid symbols are programme stars. The three stars labelled are those in common with Matsuura et al. (2007). The de-reddening vector (for $A_{\mathrm{V}}=5 \mathrm{mag}$ ) is shown for two stars: it depends on the spectral type and is small for the molecular index measurements.

by Mauron et al. (2004). The chemical natures and luminosities of the detected AGB stars are compatible with current stellar evolution models, combined with the star formation histories of the host galaxies, with some fine tuning required for the extreme C star of Sculptor and for the faintest C star found in Fornax. As usual for AGB stars, a variability study would be useful to characterise the sources more completely.

Among the observed stars with $(J-K)>1.5$, we have not found any O-rich star. In other words, we have not detected new heavily dust-enshrouded M-type AGB stars. Carbon stars can have a $(J-K)$ index of about 2 even without circumstellar dust. We find eight carbon stars with $(J-K)>2$ (7 in Fornax, 1 in
Sculptor), which are most likely all dust-enshrouded. The absence of O-rich dust-enshrouded AGB stars in our sample may be the random result of small numbers; but it is not too surprising a result considering the large range of initial masses for which the final AGB stages are carbon rich at sub-solar metallicities. The low mass AGB stars $\left(<1 M_{\odot}\right)$ spend a very short fraction of their final O-rich AGB life in phases of heavy mass loss and are also less luminous than their higher mass counterparts; the most massive AGB stars spend a larger fraction of their final O-rich AGB life in dust but are intrinsically rare due to the stellar IMF.

$J$ and $H$-band spectra of carbon stars are still rather rare in the literature. Our data confirms the well-behaved correlation 
between the two main bands of $\mathrm{C}_{2}$, near $1.15 \mu \mathrm{m}$ and $1.77 \mu \mathrm{m}$. In some of our spectra, the $1.53 \mu \mathrm{m}$ feature probably due to $\mathrm{C}_{2} \mathrm{H}_{2}$ is extremely deep. This feature would deserve more attention, as it is remains difficult to reproduce with models. From our small sample, it seems that $(J-K)>2$ is a requirement for the presence of a strong $1.53 \mu \mathrm{m}$ feature, but is not a sufficient condition. When the $1.53 \mu \mathrm{m}$ feature is strong, $\mathrm{C}_{2}$ bands are weak, as expected from chemistry in sufficiently cool and dense layers. All this suggests that the $1.53 \mu \mathrm{m}$ feature is related to the processes that drive mass loss from carbon stars. Confirmation of the trends from repeated observations and a larger sample will be welcome.

Acknowledgements. The authors would like to thank Peter Hauschildt and Joachim Puls for providing the high-resolution B-star model spectra in the infrared. Drs. Whitelock and Menzies are thanked for checking some stars on their IRTF images. Drs. Yves Yung and Wolfgang Hummel (both ESO) are thanked for introducing the ISAAC pipeline. The telluric spectrum was taken based on NSO/Kitt Peak FTS data produced by NSF/NOAO. This research has made use of the SIMBAD database, operated at CDS, Strasbourg, France.

\section{References}

Aaronson, M., \& Mould, J. 1980, ApJ, 240, 804

Aaronson, M., Da Costa, G. S., Hartigan, P., et al. 1984, ApJ, 277, L9

Aaronson, M., Mould, J., \& Cook, K. H. 1985, ApJ, 291, L41

Azzopardi, M. 1999, Ap\&SS, 265, 291

Azzopardi, M., Lequeux, J., \& Westerlund, B. E. 1985, A\&A, 144, 388

Azzopardi, M., Muratorio, G., Breysacher, J., \& Westerlund, B. E. 1999, in The stellar content of Local Group galaxies, ed. P. Whitelock, \& R. Cannon (ASP: San Francisco), IAU Symp., 192, 144

Babusiaux, C., Gilmore, G., \& Irwin, M. 2005, MNRAS, 359, 985

Battinelli, P., \& Demers, S. 2005a, A\&A, 434, 657

Battinelli, P., \& Demers, S. 2005b, A\&A, 442, 159

Bersier, D., \& Wood, P. R. 2002, AJ, 123, 840

Bessell, M. S., \& Wood, P. R. 1984, PASP, 96, 247

Buonanno, R., Corsi, C. E., Zinn, R., et al. 1998, ApJ, 501, L33

Cardelli, J. A., Clayton, G. C., \& Mathis, J. S. 1989, ApJ, 345, 245

Castelli, F., Gratton, R. G., \& Kurucz, R. L. 1997, A\&A, 318, 841

Cioni, M.-R. L., \& Habing, H. J. 2005b, A\&A, 429, 837

Cioni, M.-R. L., \& Habing, H. J. 2005a, A\&A, 442, 165

Clementini, G., Held, E. V., Baldacci, L., \& Rizzi, L. 2003, ApJ, 588, 85

Clementini, G., Ripepi, V., Bragaglia, A., et al. 2005, MNRAS, 363, 734

Coleman, M., Da Costa, G. S., Bland-Hawthorn, J., \& Freeman, K. C. 2005, AJ, 129,1443

Cook, K. H., \& Aaronson, M. 1989, AJ, 97, 923

Cushing, M. C., Rayner, J. T., \& Vacca, W. D. 2005, ApJ, 623, 1115

Cutri, R. M., Skrutskie, M. F., Van Dyk, S., et al. 2003, Explanatory Supplement to the 2MASS All-Sky Data Release

Davidge, T. J. 2005, AJ, 130, 2087

de Blok, W. J. G., \& Walter, F. 2006, AJ, 131, 343

Dejonghe, H., \& Van Caelenberg, K. 1999, in asymptotic giant branch Stars, ed.

T. Le Bertre, A. Lèbre, \& C. Waelkens (San Francisco: ASP), IAU Symp., 191,501

Demers, S., \& Kunkel, W. E. 1979, PASP, 91, 761

Demers, S., Battinelli, P., \& Artigau, E. 2006, A\&A, 456, 905

Demers, S., Battinelli, P., \& Kunkel, W. E. 2006, A\&A, 636, L85

Elias, J. H., \& Frogel, J. A. 1985, ApJ, 289, 141

Fluks, M. A., Plez, B., Thé, P. S., et al. 1994, A\&AS, 105, 311

Frémaux, J., Kupka, F., Boisson, C., Joly, M., \& Tsymbal, V. 2006, A\&A, 449, 109

Gallart, C., Aparicio, A., Bertelli, G., \& Chiosi, C. 1996a, AJ, 112, 1950

Gallart, C., Aparicio, A., Bertelli, G., \& Chiosi, C. 1996b, AJ, 112, 2596

Gautschy-Loidl, R., Höfner, S., Jørgensen, U. G., \& Hron, J. 2004, A\&A, 422, 289

Gieren, W., Pietrzyński, G., Nalewajko, K., et al. 2006, ApJ, 647, 1054

Goebel, J. H., Bregman, J. D., Witteborn, F. C., Taylor, B. J., \& Willner, S. P. 1981, ApJ, 246, 455

Gray, R. O. 2009, unpublished invited review at the workshop on Intermediate Mass vs. Massive Stars, Strasbourg Observatory, France, February 9 Groenewegen, M. A. T. 1995, A\&A, 293, 463
Groenewegen, M. A. T. 2006, in Planetary Nebulae beyond the Milky Way (ESO-Garching, May 2004), ed. L. Stanghellini, J. Walsh, \& N. G. Douglas (Berlin: Springer-verlag), 108

Groenewegen, M. A. T. 2007, in Why Galaxies care about AGB stars, ed. F. Kerschbaum, C. Charbonnel, \& B. Wing, ASPC Ser., 378, 433

Groenewegen, M. A. T., Wood, P. R., Sloan, G. C., et al. 2007, MNRAS, 376, 313

Gullieuszik, M., Veld, E. V., Rizzi, L., et al. 2007, A\&A, 467, 1025

Habing, H. J. 1996, A\&AR, 7, 97

Harbeck, D., Gallagher, J. S III, \& Grebel, E. K. 2004, AJ, 127, 2711

Joyce, R. R. 1998, AJ, 115, 2059

Joyce, R. R., Hinkle, K. H., Wallace, L., Dulick, M., \& Lambert, D. L. 1998, AJ, 116,2520

Kang, A., Sohn, Y.-J., Rhee, J., et al. 2005, A\&A, 437, 61

Kang, A., Sohn, Y.-J., Kim, H.-I., et al. 2006, A\&A, 454, 717

Kerschbaum, F., Charbonnel, C., \& Wing, R. F. 2007, Why Galaxies Care about AGB Stars: their Importance as Actors and Probes, ASP Conf. Ser., 378

Lagadec, E., Zijlstra, A. A., Matsuura, M., et al. 2008, MNRAS, 383, 399

Lançon, A. 1999, in Asymptotic Giant Branch Stars, ed. T. Le Bertre, A. Lèbre, \& C. Waelkens (San Francisco: ASP), IAU Symp., 191, 579

Lançon, A., \& Wood, P. R. 2000, A\&AS, 146, 217 (LW2000)

Lançon, A., Mouhcine, M., Fioc, M., \& Silva, D. 1999, A\&A, 344, L21

Lançon, A., Hauschildt, P. H., Ladjal, D., \& Mouhcine, M. 2007, A\&A, 468, 205

Letarte, B., Demers, S., Battinelli, P., \& Kunkel, W. E. 2002, AJ, 123, 832

Maillard, J. P., Chauville, J., \& Mantz, A. W. 1976, J. Molec. Spectrosc., 63, 120

Marigo, P., \& Girardi, L. 2007, A\&A, 469, 239

Marigo, P., Girardi, L., Bressan, A., et al. 2008, A\&A, 482, 883

Mateo, M. 1998, ARA\&A 36, 435

Matsuura, M., Zijlstra, A. A., Bernard-Salas, J., et al. 2007, MNRAS, 382, 1889 Mauron, N., Azzopardi, M., Gigoyan, K., \& Kendall, T. R. 2004, A\&A, 418, 77 McLean, I. S., McGovern, M. R., Burgasser, A. J., et al. 2003, ApJ, 596, 561

Menzies, J., Feast, M., Tanabé, T., Whitelock, P., \& Nakada, Y. 2002, MNRAS, 335,923

Moorwood, A., Cuby, J.-G., Biereichel, P., et al. 1998, The Messenger, 94, 7

Morris, R. A. H., Phillipps, S., Jones, J. B., et al. 2007, A\&A, 476, 59

Mouhcine, M. 2002, A\&A, 394, 125

Mouhcine, M., \& Lançon, A. 2003, MNRAS, 338, 572

Nowotny, W., \& Kerschbaum, F. 2002, Hvar Obs. Bulletin, 26, 63

Nowotny, W., Kerschbaum, F., Schwarz, H. E., \& Olofsson, H. 2001, A\&A, 367, 557

Nowotny, W., Kerschbaum, F., Olofsson, H., \& Schwarz, H. E. 2003, A\&A, 403, 93

Origlia, L., Moorwood, A. F. M., \& Oliva, E. 1993, A\&A, 280, 536

Palmer, L. G., \& Wing, R. F. 1982, AJ, 87, 1739

Pietrzyński, G., Gieren, W., Szewczyk, O., et al. 2008, AJ, 135, 1993

Pont, F., Zinn, R., Gallart, C., Hardy, E., \& Winnick, R. 2004, AJ, 127, 840

Richer, H. B., Crabtree, D. R., \& Pritchet, C. J. 1984, ApJ, 287, 138

Rizzi, L. 2002, Ph.D. Thesis, University of Padua

Rizzi, L., Held, E. V., Saviane, I., Tully, R. B., \& Gullieuszik, M. 2007, MNRAS, 380,1255

Rowe, J. F., Richer, H. B., Brewer, J. P., \& Crabtree, D. R. 2005, AJ, 129, 729

Saviane, I., Held, E. V., \& Bertelli, G. 2000, A\&A, 355, 56

Schweitzer, A. E., Cudworth, K. M., Majewski, S. R., \& Suntzeff, N. B. 1995, AJ, 110, 2747

Schlegel, D. J., Finkbeiner, D. P., \& Davis, M. 1998, ApJ, 500, 525

Sohn, Y.-J., Kang, A., Rhee, J., et al. 2006, A\&A, 445, 69

Stetson, P. B., Hesser, J. E., \& Smecker-Hane, T. A. 1998, PASP, 110, 533

Tej, A., Lançon, A., Scholz, M., \& Wood, P. R. 2003, A\&A, 412,481

Terndrup, D. M., Frogel, J. A., \& Whitford, A. E. 1991, ApJ, 378, 742

Tolstoy, E., Venn, K. A., Shetrone, M., et al. 2003, AJ, 125, 707

Tolstoy, E., Irwin, M. J., Helmi, A., et al. 2004, ApJ, 617, L119

Trams, N. R., van Loon, J. Th., Waters, L. B. F. M., et al. 1999, A\&A, 346, 843

van Loon, J. Th., Groenewegen, M. A. T., de Koter, A., et al. 1999, A\&A, 351, 559

van Loon, J. Th., Marshall, J. R., Cohen, M., et al. 2006, A\&A, 447, 971

van Loon, J. Th., Cohen, M., Oliveira, J. M., et al. 2008, A\&A, 487, 1055

Venn, K. A., Lennon, D. J., Kaufer, A., et al. 2001, ApJ, 547, 765

Westerlund, B. E., Edvardsson, B., \& Lindgren, K. 1987, A\&A, 178, 41

Whitelock, P. A., Feast, M. W., van Loon, J. Th., \& Zijlstra, A. A. 2003, MNRAS, 342, 86

Whitelock, P. A., Feast M. W., Marang, F., \& Groenewegen, M. A. T. 2006, MNRAS, 369, 751

Whitelock, P. A., Menzies, J. W., Feast, M. W., et al. 2009, MNRAS, 394, 795

Wing, R. F. 1971, in Late-Type Stars, ed. G. W. Lockwood, \& H. M. Dyck, Kitt

Peak National Observatory, Contribution, 554, 145

Wyder, T. K. 2003, AJ, 125, 3097 
M. A. T. Groenewegen et al.: Near-infrared spectroscopy of AGB star candidates, Online Material p 1
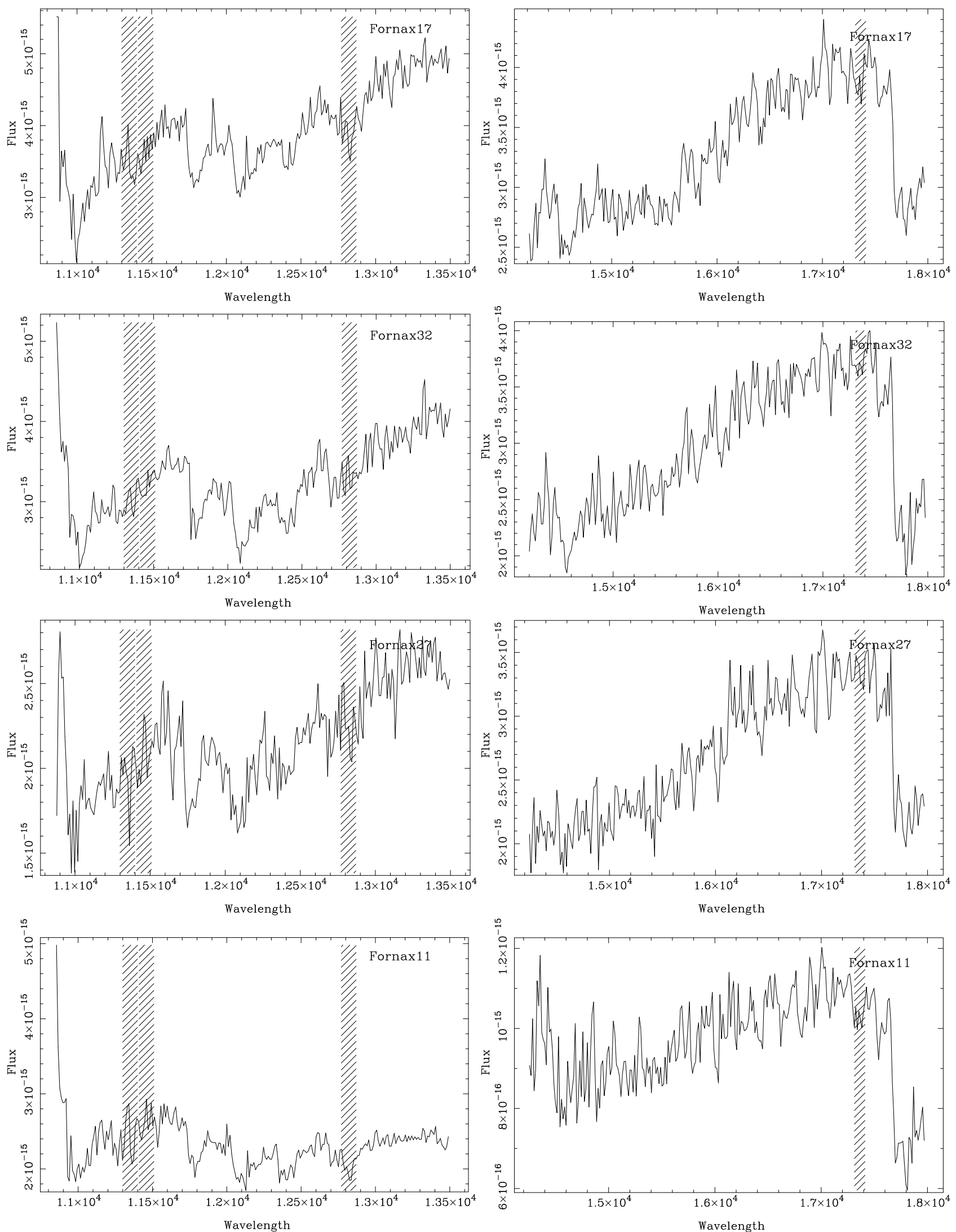

Fig. 6. $J$ and $H$-band spectra obtained with ISAAC. Wavelengths are in $\AA$ fluxes in $\mathrm{W} / \mathrm{m}^{2} / \mu \mathrm{m}$. Shaded areas indicate wavelength regions where the cancellation of the atmospheric features and/or of features in the standard stars may be imperfect. The carbon stars in Fornax are presented first, ordered by increasing $(J-K)$ colour, followed by the one red carbon star in Sculptor. Subsequently the O-stars are plotted by galaxy, and by increasing $(J-K)$ colour. Finally, for completeness, we append the spectra of previously known sources (observed for reference), and those of outliers (source 16 in Fornax for which our $\mathrm{H}$-band spectrum is odd, as well as the confirmed or likely low-redshift galaxies). 
M. A. T. Groenewegen et al.: Near-infrared spectroscopy of AGB star candidates, Online Material p 2
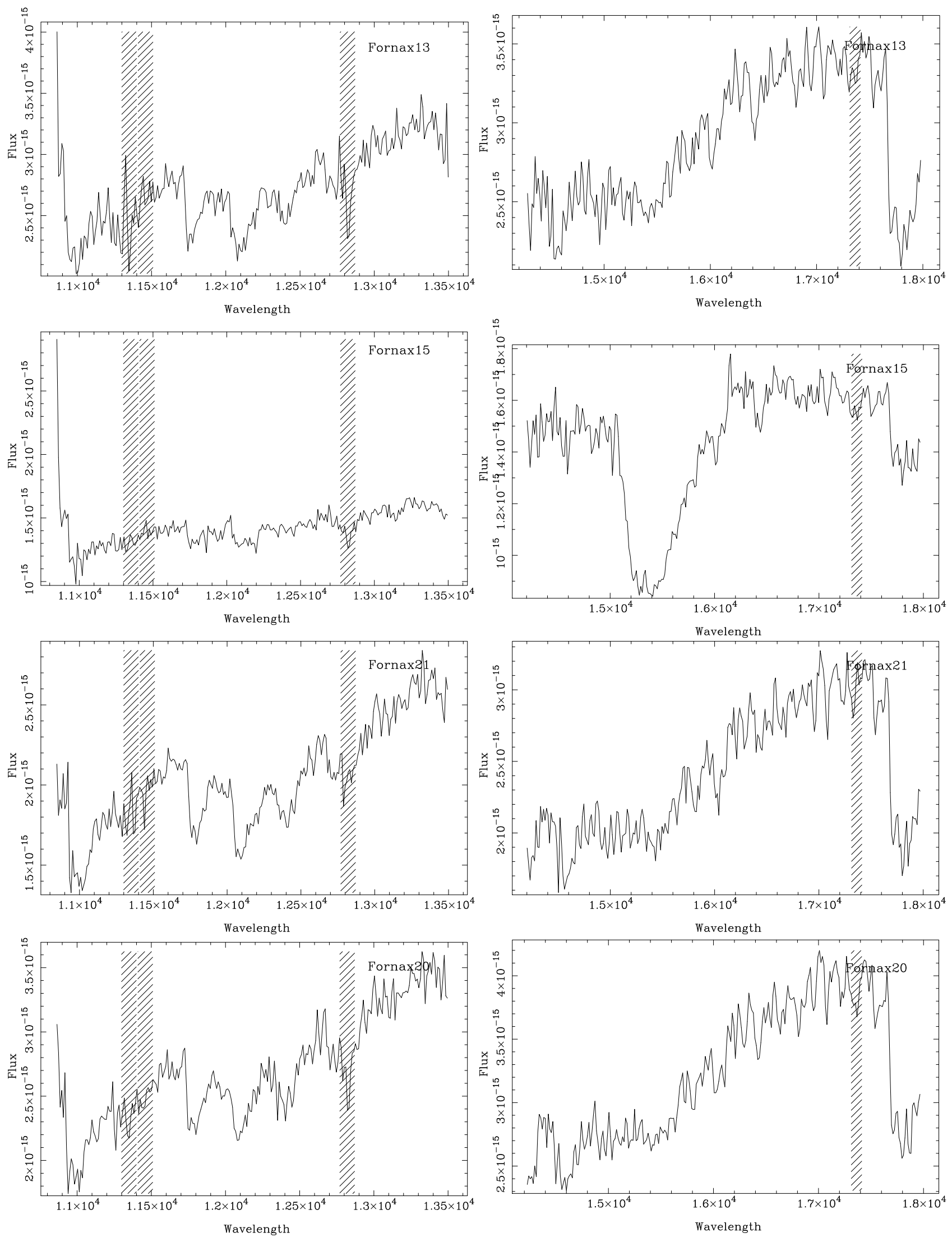

Fig. 6. continued. 
M. A. T. Groenewegen et al.: Near-infrared spectroscopy of AGB star candidates, Online Material p 3
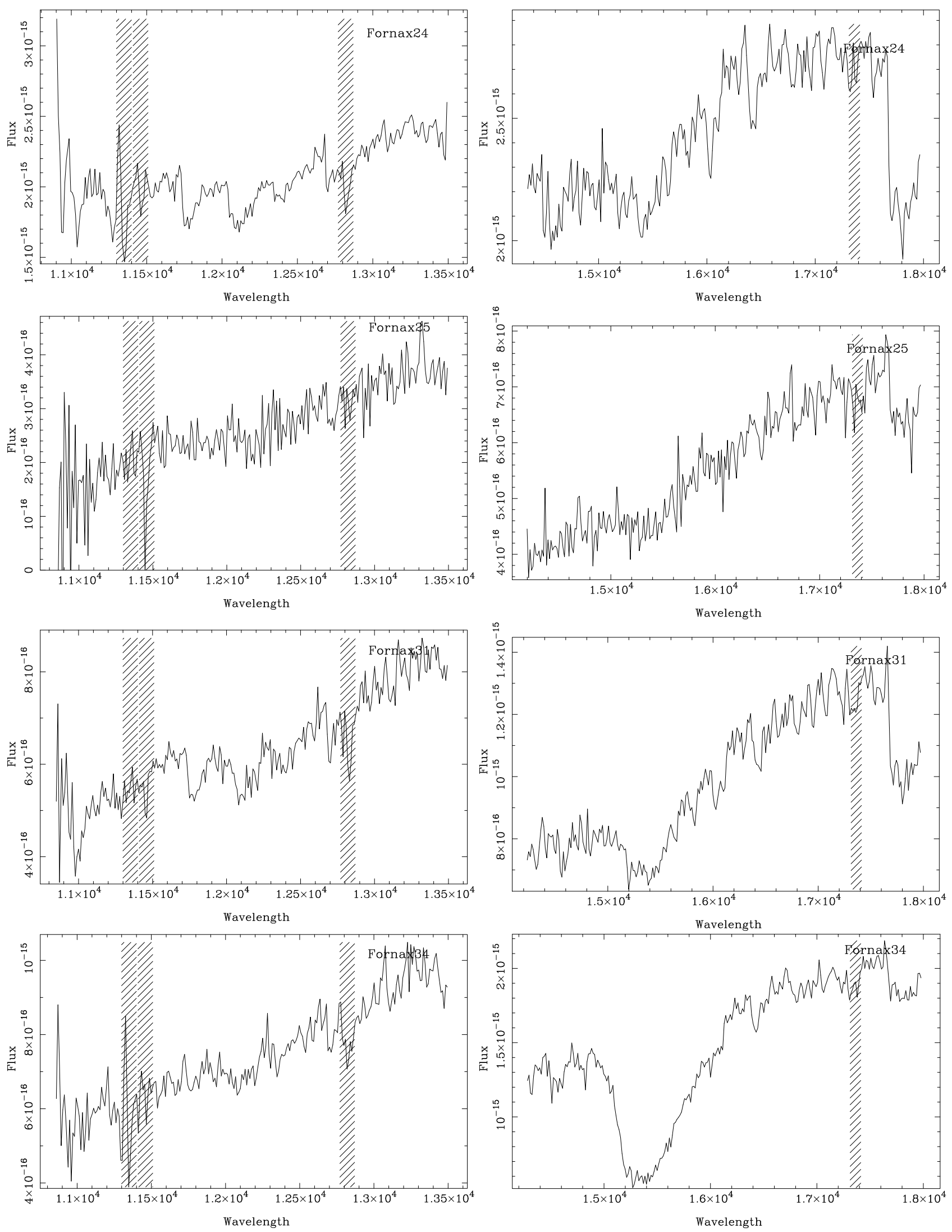

Fig. 6. continued. 
M. A. T. Groenewegen et al.: Near-infrared spectroscopy of AGB star candidates, Online Material p 4
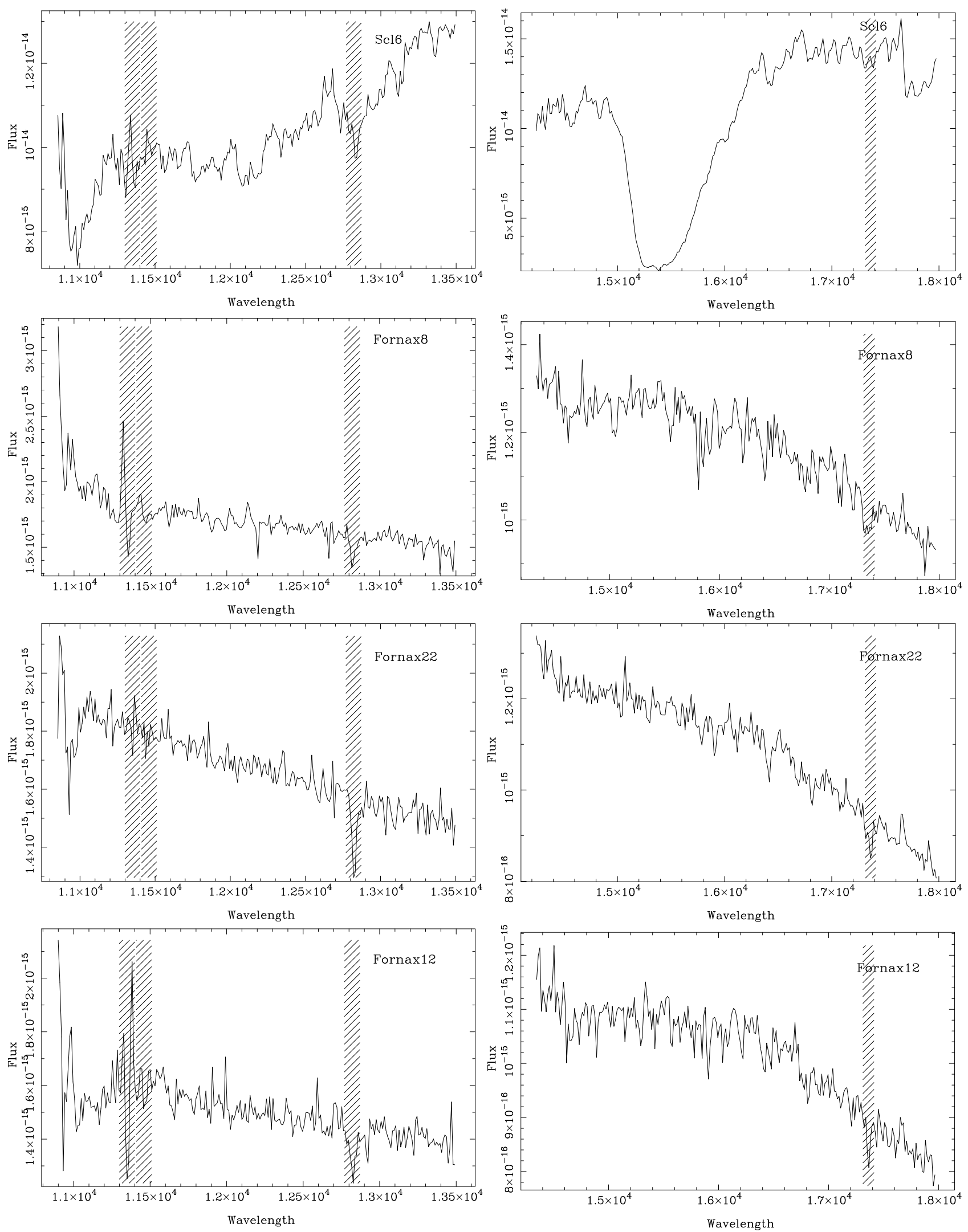

Fig. 6. continued. 
M. A. T. Groenewegen et al.: Near-infrared spectroscopy of AGB star candidates, Online Material p 5
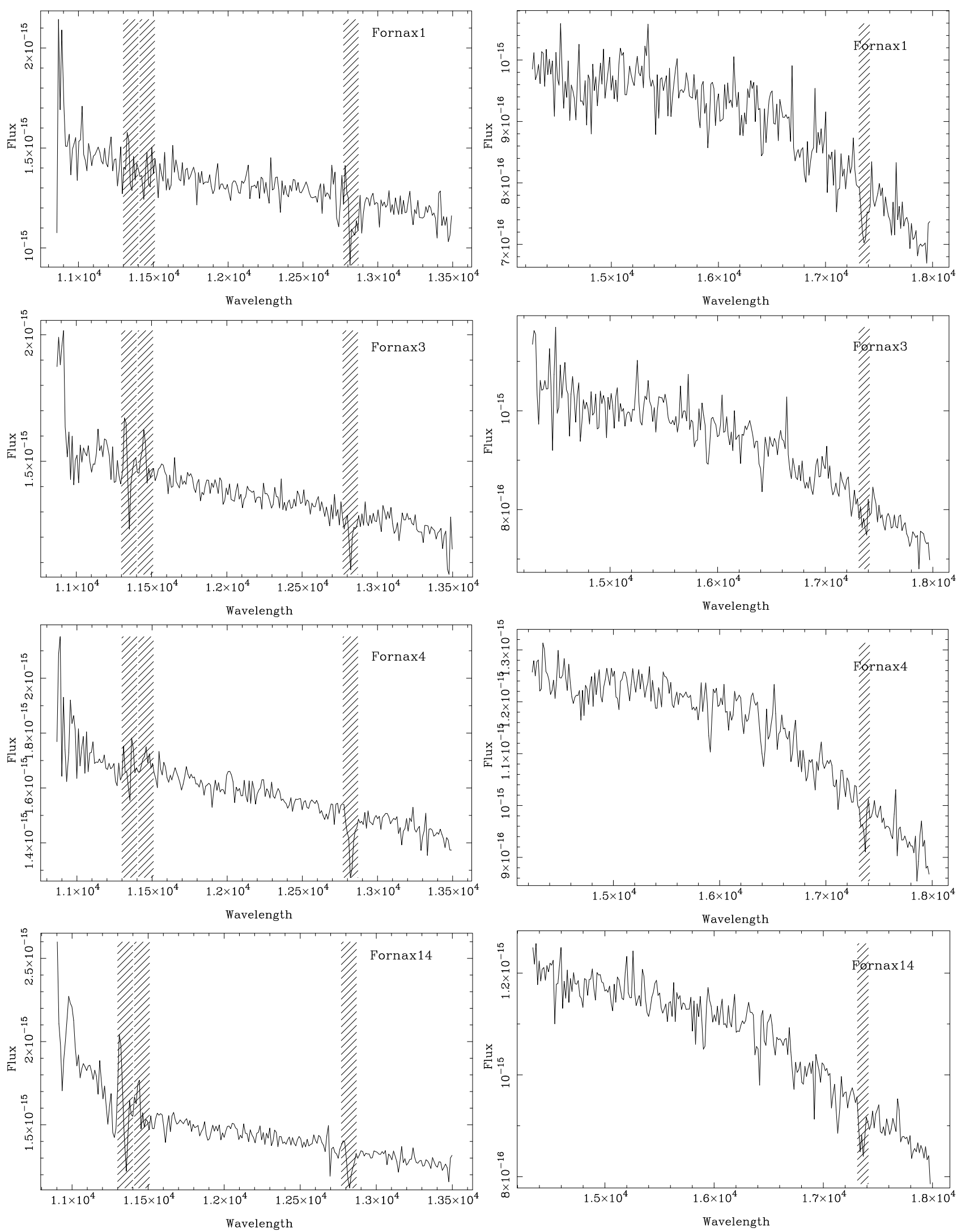

Fig. 6. continued. 
M. A. T. Groenewegen et al.: Near-infrared spectroscopy of AGB star candidates, Online Material p 6
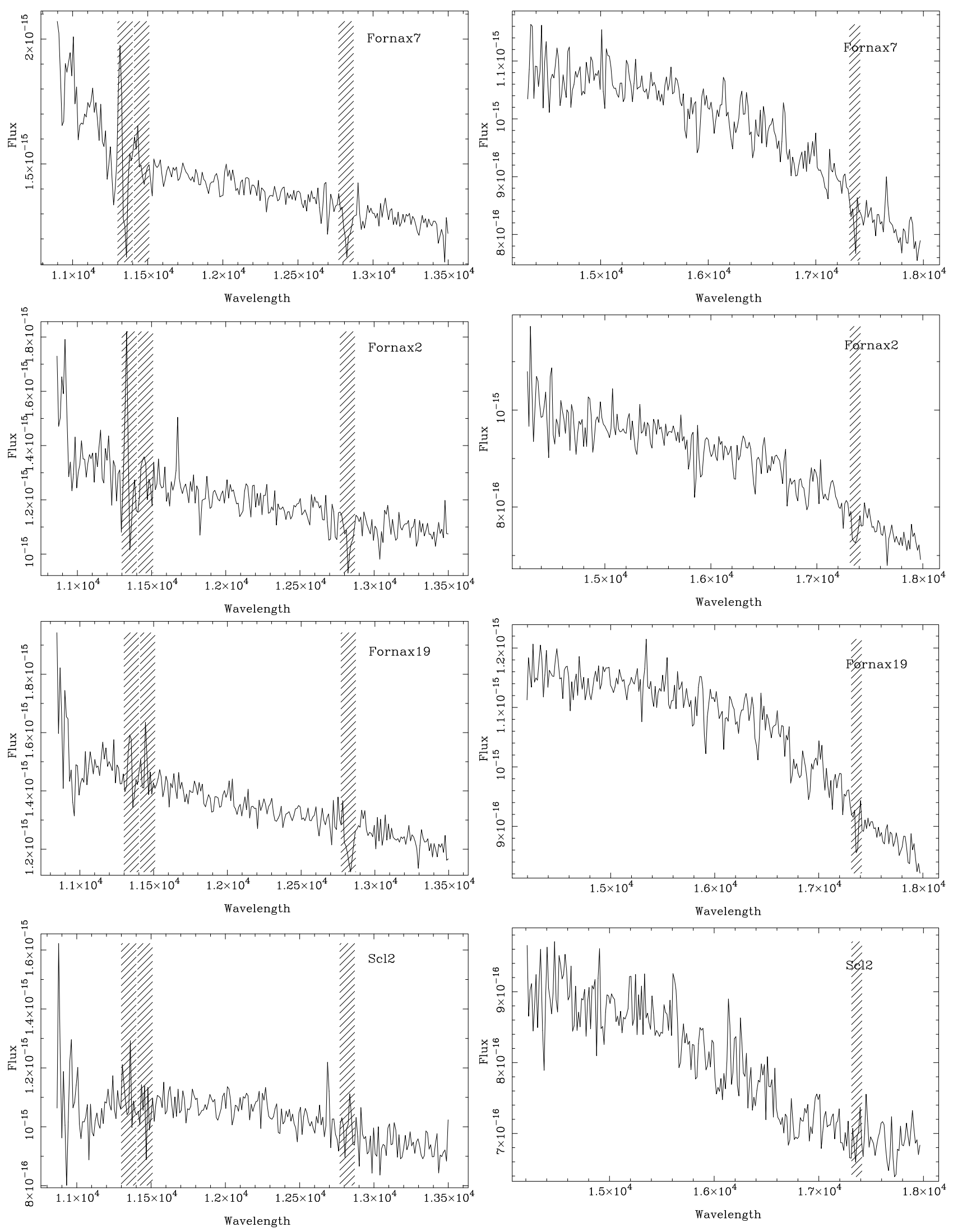

Fig. 6. continued. 
M. A. T. Groenewegen et al.: Near-infrared spectroscopy of AGB star candidates, Online Material p 7
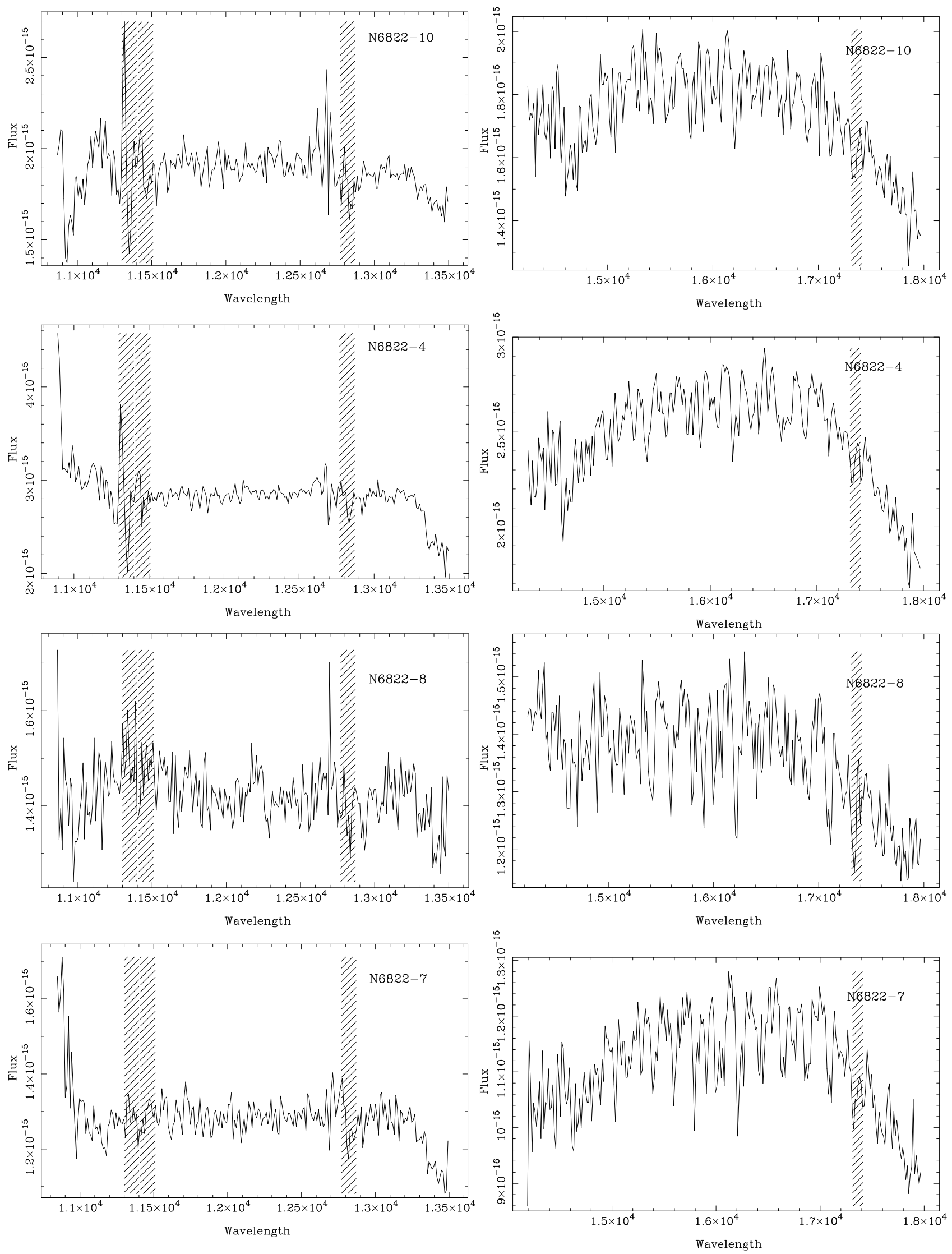

Fig. 6. continued. 
M. A. T. Groenewegen et al.: Near-infrared spectroscopy of AGB star candidates, Online Material p 8
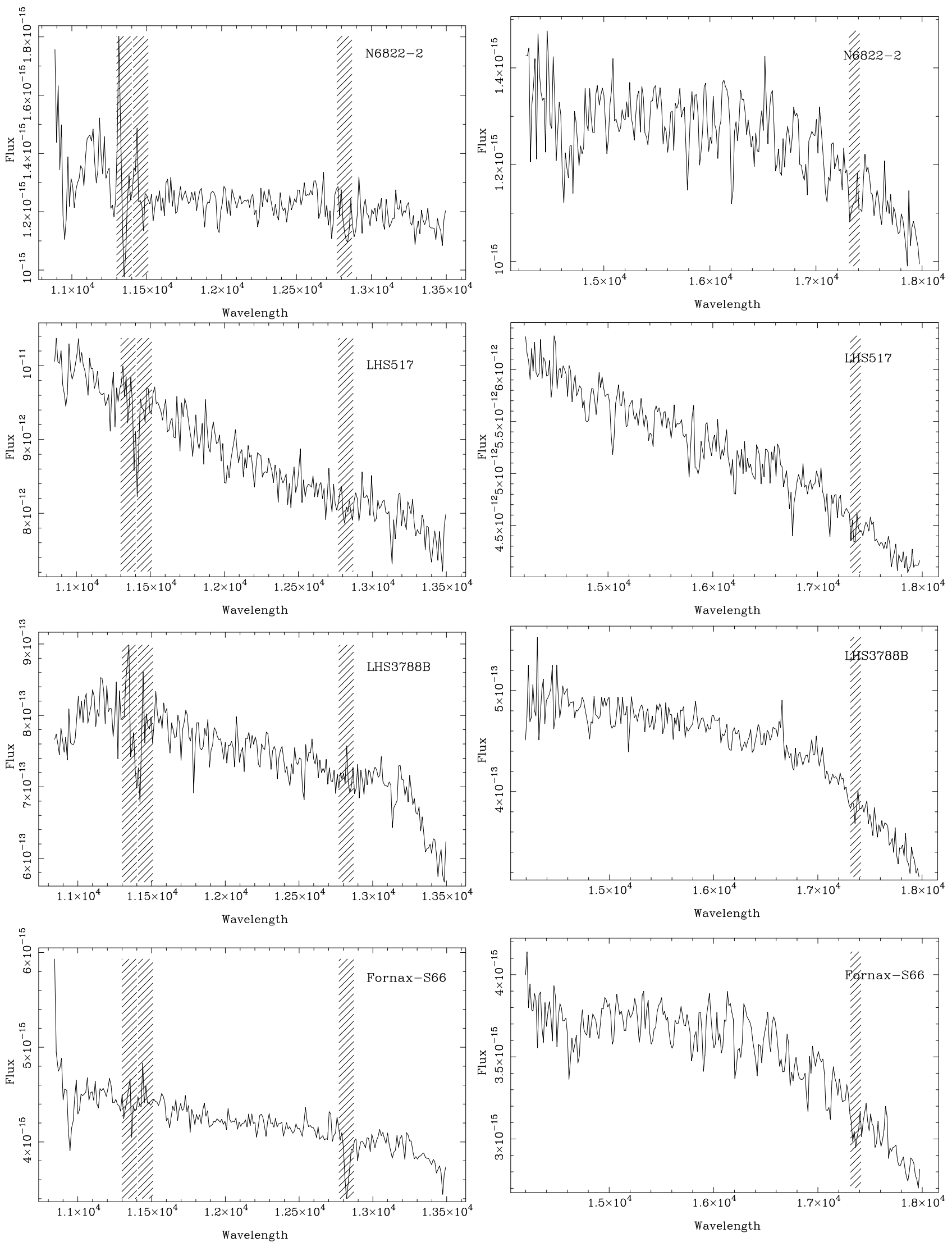

Fig. 6. continued. 
M. A. T. Groenewegen et al.: Near-infrared spectroscopy of AGB star candidates, Online Material p 9
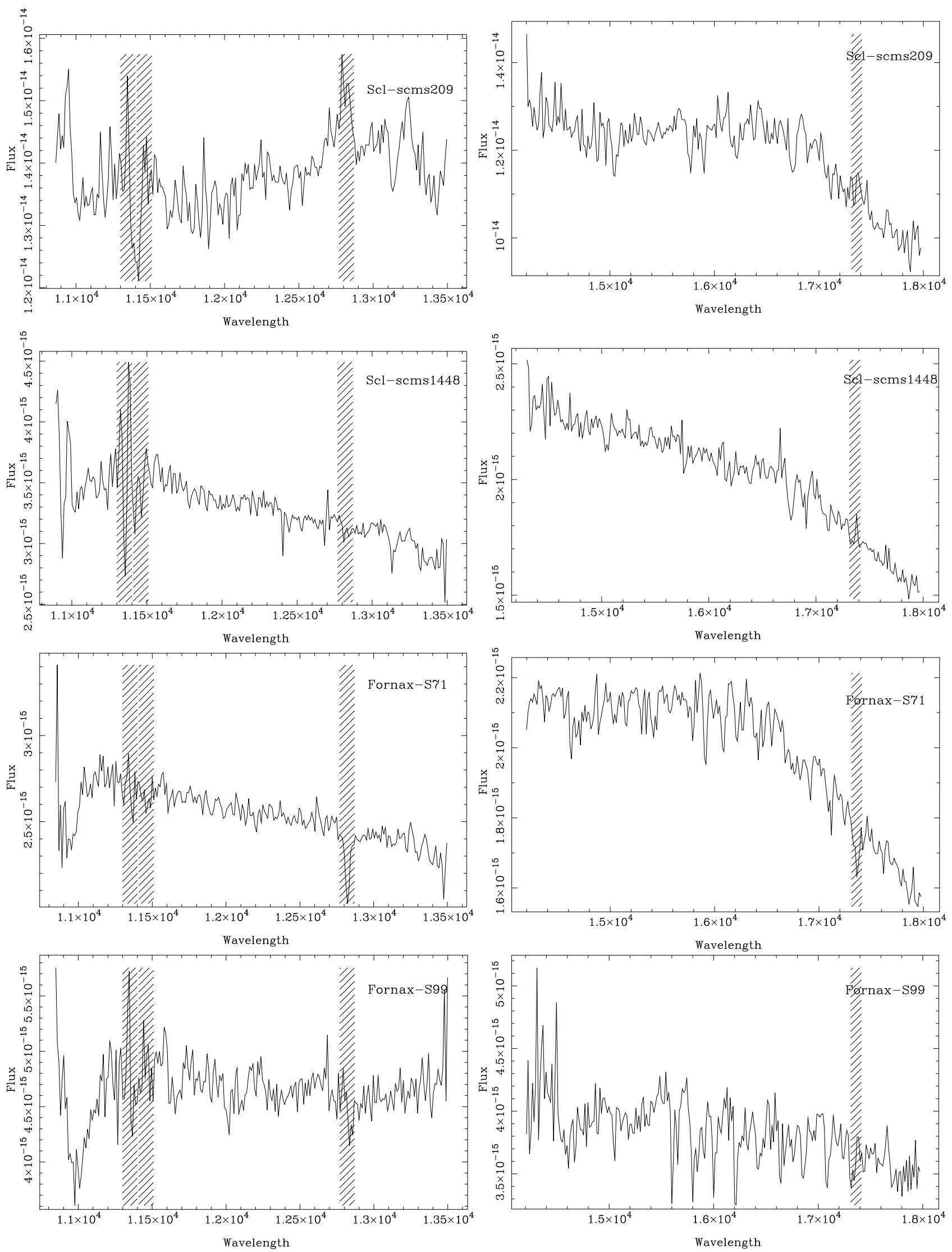

Fig. 6. continued. 
M. A. T. Groenewegen et al.: Near-infrared spectroscopy of AGB star candidates, Online Material p 10
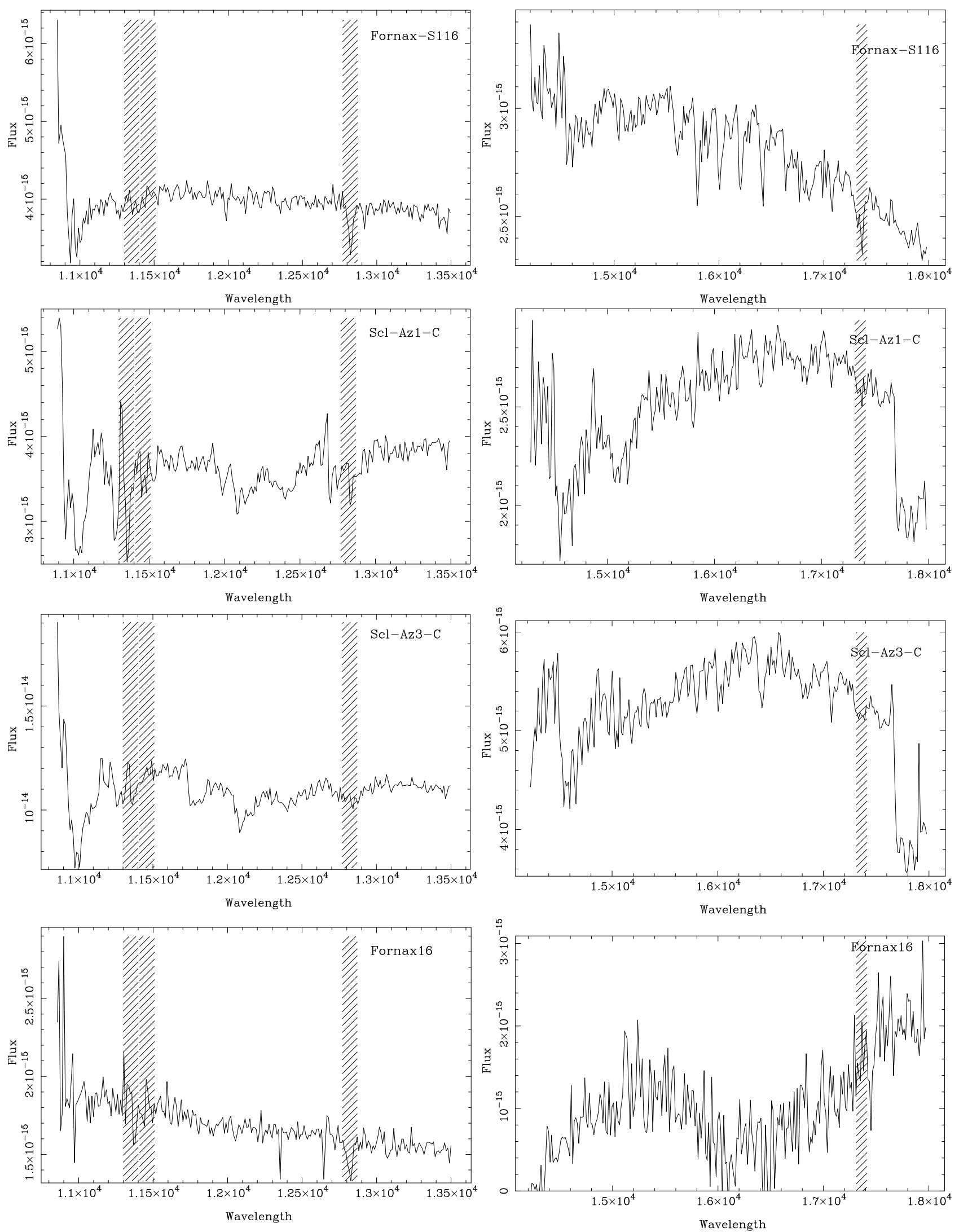

Fig. 6. continued. 
M. A. T. Groenewegen et al.: Near-infrared spectroscopy of AGB star candidates, Online Material p 11
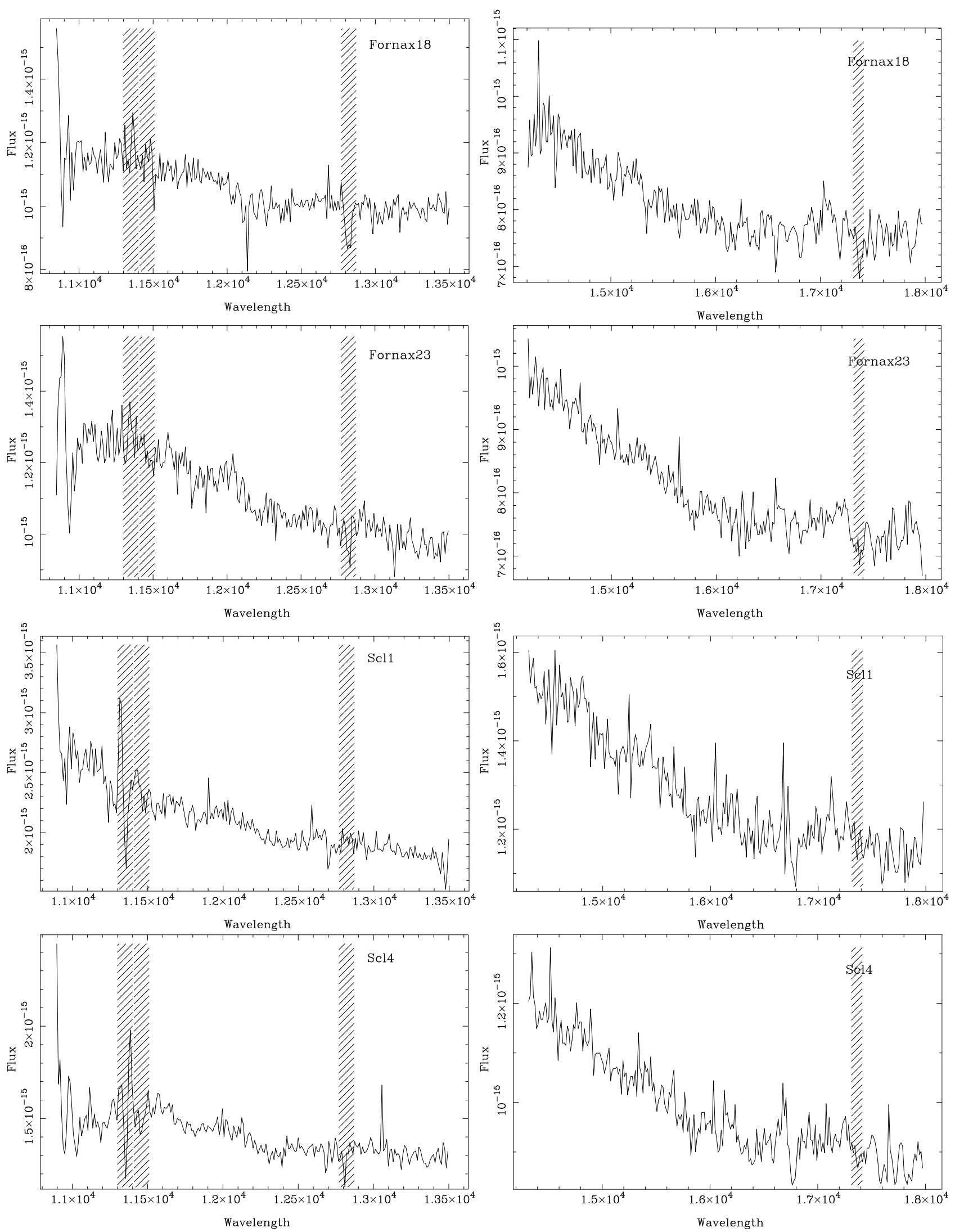

Fig. 6. continued. 
M. A. T. Groenewegen et al.: Near-infrared spectroscopy of AGB star candidates, Online Material p 12
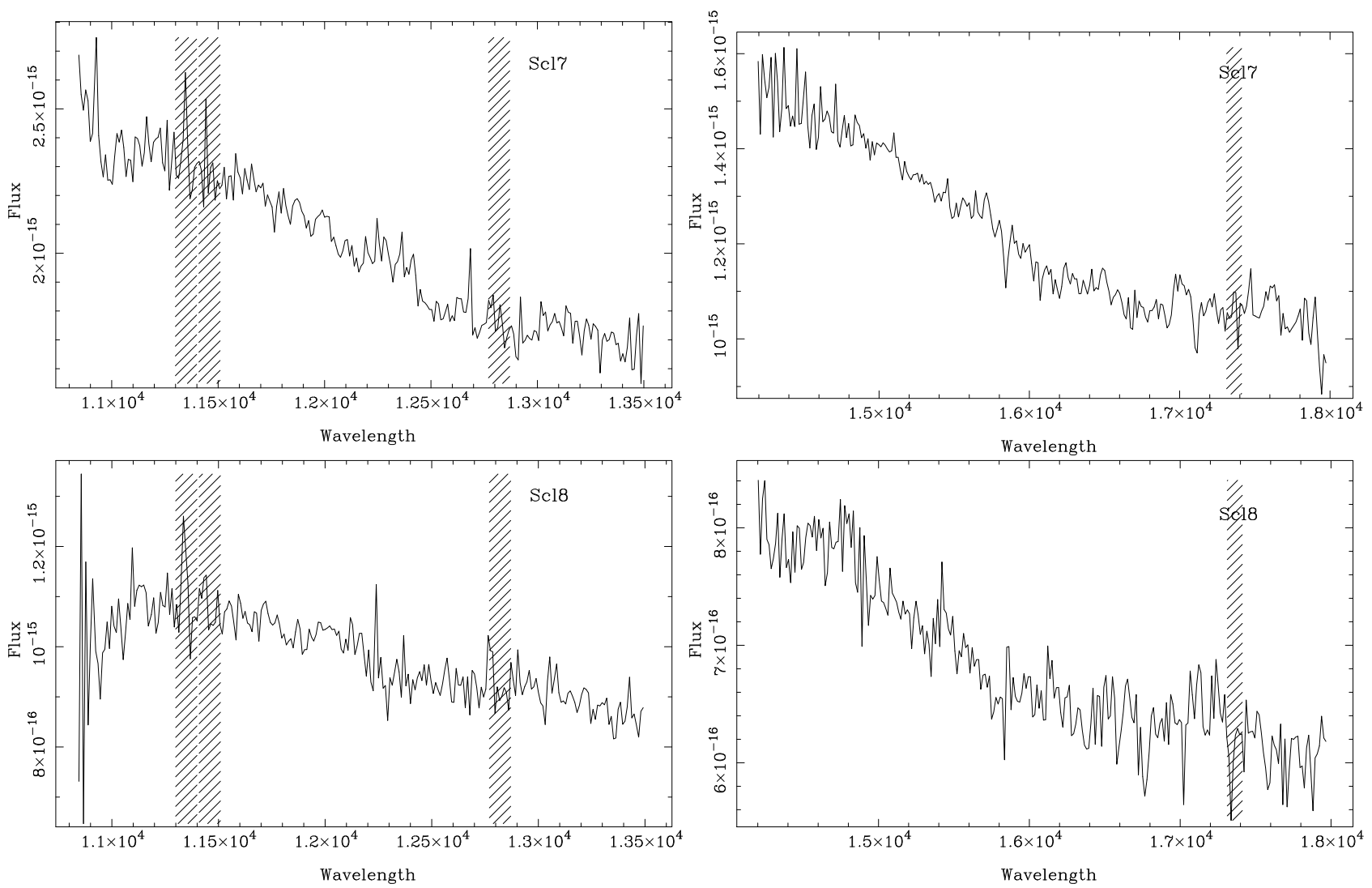

Fig. 6. continued. 